\title{
Capture into slow-invariant-manifold in the fluid-structure dynamics of a sprung cylinder with a nonlinear rotator
}

\author{
Antoine B. Blanchard ${ }^{\mathrm{a}, *}$, Oleg V. Gendelman ${ }^{\mathrm{b}}$, \\ Lawrence A. Bergman ${ }^{\mathrm{a}}$, Alexander F. Vakakis ${ }^{\mathrm{c}}$ \\ ${ }^{a}$ Department of Aerospace Engineering, University of Illinois at Urbana-Champaign, \\ 104 South Wright Street, Urbana, IL 61801, United States \\ ${ }^{b}$ Faculty of Mechanical Engineering, Technion, Israel Institute of Technology, \\ Haifa 32000, Israel \\ ${ }^{c}$ Department of Mechanical Science and Engineering, University of Illinois at Urbana-Champaign, \\ 1206 West Green Street, Urbana, IL 61801, United States
}

\begin{abstract}
We investigate the dynamics of a two-dimensional circular cylinder mounted on a linear spring, restricted to move in the cross-flow direction and undergoing vortex-induced vibration, incorporating a strongly nonlinear (i.e., non-linearizable) internal element consisting of a mass that is free to rotate about the cylinder axis and whose angular motion is restrained by a linear viscous damper. The conjunction of the essentially nonlinear inertial coupling with the dissipative element makes the internal attachment behave as a nonlinear energy sink that is able to extract and dissipate energy from the motion of the cylinder and (indirectly) the surrounding fluid. At the intermediate Reynolds number $R e=100$, we find that the cylinder with rotator undergoes repetitive cycles of slowly decaying oscillations interrupted by chaotic bursts; during the slowly decaying portion of each cycle, the dynamics of the cylinder is regular and can lead to significant vortex street elongation with partial stabilization of the wake. We construct a reduced-order model of the fluid-structure interaction dynamics based on the data obtained by direct numerical simulation, and employ analytical techniques such as complexification/averaging and the multiple-scales method to show that the strongly modulated response is the manifestation of a resonance capture into a slow invariant manifold (SIM) that leads to targeted energy transfer from the cylinder to the rotator. Capture into the SIM corresponds to transient cylinder stabilization, whereas escape from the SIM leads to chaotic bursts. Hence, the action of the nonlinear rotator on the resonance dynamics of the fluid-structure interaction is clarified.
\end{abstract}

Keywords: vortex-induced vibrations, nonlinear energy sink, resonance capture, slow invariant manifold

\footnotetext{
*Corresponding author

Email address: ablancha@illinois.edu (Antoine B. Blanchard)
} 


\section{Introduction}

Flow separation aft of an elastically-supported bluff body results in shedding of vortices, thus generating an oscillating lift force that excites the body in such a way that it undergoes large amplitude vortex-induced vibrations (VIV) $[6,7,38]$. Structures that are subjected to VIV can be found in a broad range of engineering applications; e.g., tall chimneys, power transmission lines and off-shore structures. In most practical cases, suppression of VIV achieved using passive or active approaches is sought in order to reduce or even completely eliminate excessive vibrations and dynamic instabilities $[3,9,30]$. In other cases, for instance in the context of energy harvesting and power pick-off, the effects of VIV can be beneficial, since it can be incorporated into designs to achieve efficient harvesting in flow-structure interaction regimes. In such applications, focus is directed toward enhancing the flow-induced vibrations in order to achieve large-amplitude motions that, in turn, may have implications for efficient energy harvesting [4, 5, 10, 20, 21].

A prototypical VIV model consists of the flow past a circular cylinder mounted on a grounded linear spring, and constrained to move in the cross-flow direction. Recent studies have investigated the dynamics of a system in which the dimensionality of the standard VIV configuration is augmented by coupling the cylinder with an essentially nonlinear dissipative attachment (or nonlinear energy sink, NES), which is free to translate and/or rotate inside the cylinder (with no gravitational effects) [33-35]. The NES, because it features a dissipative element and strong inertial nonlinearity, has the capacity to absorb vibrational energy from the primary structure to which it is attached, over broad frequency and energy ranges and for various types of narrowband or broadband excitations. For instance, it has been applied for passively mitigating aeroelastic instabilities in an aircraft wing [18]. Moreover, the one-way, almost irreversible, nonlinear targeted energy transfer (TET) from a primary structure to the NES is related to cascades of transient resonance captures on slowly-invariant resonance manifolds [2, 31, 32, 37].

The addition of a rotational NES to the transverse VIV model has been shown to result in drastic passive suppression of vibrations of the cylinder at intermediate Reynolds number $(60 \leq R e \leq 120)$ [33]. Although there is no direct interaction between the NES and the infinite-dimensional flow, the rotational attachment has an indirect effect on the flow, i.e., through the dynamics of the cylinder to which it is inertially coupled [36]. One notable VIV suppression mechanism of particular interest that has been identified consists of repetitive cycles of slowly decaying oscillations of the cylinder followed by intermediate chaotic bursts [36]. This interesting nonlinear dynamical phenomenon has been attributed to the presence of the rotating internal NES, since no similar results have been found for the case of the translational NES [33, 34]. Moreover, during the slowly decaying part of the cycle, the rotational NES performs phase-locked steady rotations, and this is accompanied by a noticeable reduction of the lift and drag coefficients of the cylinder, as well as profound effects on the wake; in particular, vortex street elongation occurs, resulting in partial stabilization of the wake behind the cylinder.

In this work, we aim to show that the repetitive cycles of slowly decaying cylinder motions interrupted by chaotic bursts result from successive resonance captures on and escapes from a slow invariant manifold of the dynamics, during which the cylinder, the pendulum and the flow - meaning, the lift force — are locked into a regime of 1:1:1 resonance, thus leading to targeted energy transfer from the surrounding fluid and the oscillating cylinder to the NES. We first perform an extensive series of direct numerical 
simulations (DNS) to identify sets of the NES parameters for which such cycles are realized at $R e=100$, and observe that this behavior is found over a relatively broad range of NES parameters. Next, we analytically study the dynamics of the system in the regime of the aforementioned resonance where the succession of slowly decaying cycles occurs. In our asymptotic analysis, we focus on cases where the ratio of the NES mass to that of the sprung cylinder is either small or finite. For small values of this mass ratio, we show that, after proper complexification/averaging of the equations of motion, the resonant fluidstructure interaction dynamics is amenable to multiple-scales analysis. For larger values of the mass ratio, however, we rely on a slow-fast partition of the dynamics to study the slowly decaying cycle. In both cases, we find good agreement between direct numerical simulations of the full-order system and the approximate analysis, thus demonstrating that the slowly decaying cycles interrupted by chaotic bursts are caused by successive resonance captures on and escapes from an underlying slow invariant manifold of the 1:1:1 resonant dynamics. The analytical results then pave the way for predictive design of the internal nonlinear element (in this case the rotator) for optimal flow stabilization.

\section{Computational model and motivation}

\subsection{Physical model and governing equations}

We consider a two-dimensional circular cylinder of mass $M_{\text {cyl }}$ and diameter $D$, immersed in an incompressible fluid of density $\rho_{\mathrm{f}}$ and kinematic viscosity $\nu$. The cylinder is mounted on a linear spring of stiffness $K_{\mathrm{cyl}}$, and allowed to move only in the direction transverse to the free stream. A pendulum of mass $M_{\mathrm{NES}}$ is attached to the cylinder and freely rotates at constant radius $r_{0}$ about the cylinder's generatrix. Inertial coupling allows energy to be transferred from the cylinder (and indirectly from the surrounding fluid) to the eccentric rotator, part of which is dissipated by a linear rotational viscous damper about the axis of rotation of the NES.

The flow is governed by the Navier-Stokes equations, which we write in dimensionless form (asterisk denoting dimensionless quantities) as

$$
\begin{aligned}
\frac{\partial \mathbf{v}^{*}}{\partial \tau}+\mathbf{v}^{*} \cdot \boldsymbol{\nabla}^{*} \mathbf{v}^{*} & =-\boldsymbol{\nabla}^{*} p^{*}+\frac{1}{R e} \boldsymbol{\nabla}^{* 2} \mathbf{v}^{*} \\
\boldsymbol{\nabla}^{*} \cdot \mathbf{v}^{*} & =0
\end{aligned}
$$

where we have defined a dimensionless time $\tau=t U / D$ and scaled the length, velocity $\mathbf{v}$ and pressure $p$ by $D, U$ and $\rho_{\mathrm{f}} U^{2} / 2$, respectively. The Reynolds number $R e=U D / \nu$ is based on the diameter of the cylinder and on the free-stream velocity.

A sketch of the system is shown in Fig. 1. We assume that the out-of-plane $z$-axis with which the cylinder generatrix is aligned - is vertical, so that gravitational effects can be ignored. The Lagrangian is written as

$$
L=\frac{1}{2}\left(M_{\mathrm{cyl}}+M_{\mathrm{NES}}\right)\left(\frac{\mathrm{d} Y}{\mathrm{~d} t}\right)^{2}+\frac{1}{2} M_{\mathrm{NES}}\left[r_{0}^{2}\left(\frac{\mathrm{d} \theta}{\mathrm{d} t}\right)^{2}-2 r_{0} \frac{\mathrm{d} Y}{\mathrm{~d} t} \frac{\mathrm{d} \theta}{\mathrm{d} t} \sin \theta\right]-K_{\mathrm{cyl}} Y^{2},
$$

where $Y$ denotes the displacement of the cylinder from its equilibrium position and $\theta$ identifies the position of the rotating mass with respect to the $y$-axis. The linear viscous 
damping coefficient of the rotational NES is denoted by $C_{\text {NES }}$ and accounted for through the Rayleigh dissipation function

$$
R_{\mathrm{diss}}=\frac{1}{2} C_{\mathrm{NES}} r_{0}^{2}\left(\frac{\mathrm{d} \theta}{\mathrm{d} t}\right)^{2} .
$$

The two-degree-of-freedom (2DOF) system (i.e., the cylinder with the internal dissipative rotator) is coupled to the flow through the lift force, $F_{\mathrm{L}}$, and the equations governing the motion of the two coupled oscillators are therefore given by

$$
\begin{aligned}
&\left(M_{\mathrm{cyl}}+M_{\mathrm{NES}}\right) \frac{\mathrm{d}^{2} Y}{\mathrm{~d} t^{2}}+K_{\mathrm{cyl}} Y=M_{\mathrm{NES}} r_{0} \frac{\mathrm{d}}{\mathrm{d} t}\left(\frac{\mathrm{d} \theta}{\mathrm{d} t} \sin \theta\right)+F_{\mathrm{L}} \\
& M_{\mathrm{NES}} r_{0}^{2} \frac{\mathrm{d}^{2} \theta}{\mathrm{d} t^{2}}+C_{\mathrm{NES}} r_{0}{ }^{2} \frac{\mathrm{d} \theta}{\mathrm{d} t}=M_{\mathrm{NES}} r_{0} \frac{\mathrm{d}^{2} Y}{\mathrm{~d} t^{2}} \sin \theta .
\end{aligned}
$$

We note that the equation governing the rotation of the NES is strongly nonlinear since the stiffness lacks a linear part, i.e., a term that is proportional to the angle $\theta$. This feature (which is common in NES designs [37]), enables resonance (and energy absorption and dissipation) of the rotator at arbitrary frequencies as it lacks a preferential resonance frequency.

Following the work of Tumkur et al. [36], we introduce the following dimensionless variables

$$
y=\frac{Y}{D}, \quad \bar{r}_{0}=\frac{r_{0}}{D}, \quad \epsilon_{\mathrm{r}}=\frac{M_{\mathrm{NES}}}{M_{\mathrm{cyl}}+M_{\mathrm{NES}}}, \quad m^{*}=\frac{\rho_{\mathrm{b}}}{\rho_{\mathrm{f}}},
$$

where $\rho_{\mathrm{b}}$ is the density of the cylinder with the NES, $m^{*}$ is a density ratio and $\epsilon_{\mathrm{r}}$ is a mass ratio. Then, the lift force is scaled in favor of a dimensionless lift coefficient defined as

$$
C_{\mathrm{L}}=\frac{2 F_{\mathrm{L}}}{\rho_{\mathrm{f}} U^{2} D}
$$

while the natural frequency of the linear spring supporting the cylinder is non-dimensionalized as

$$
\omega_{\mathrm{r}}^{* 2}=\left(2 \pi f_{n}^{*}\right)^{2}=\frac{D^{2}}{U^{2}} \frac{K_{\mathrm{cyl}}}{M_{\mathrm{cyl}}+M_{\mathrm{NES}}}
$$

and a dimensionless damping coefficient is introduced according to the relation

$$
\lambda_{\mathrm{r}}=\frac{D}{U} \frac{C_{\mathrm{NES}}}{M_{\mathrm{NES}}} .
$$

Following these normalizations, $(5,6)$ are expressed in non-dimensional form as

$$
\begin{aligned}
\ddot{y}+\omega_{\mathrm{r}}^{* 2} y & =\epsilon_{\mathrm{r}} \bar{r}_{0} \frac{\mathrm{d}}{\mathrm{d} \tau}(\dot{\theta} \sin \theta)+\frac{2 C_{\mathrm{L}}}{\pi m^{*}} \\
\ddot{\theta}+\lambda_{\mathrm{r}} \dot{\theta} & =\frac{\ddot{y}}{\bar{r}_{0}} \sin \theta
\end{aligned}
$$

where $(\dot{)})$ denotes differentiation with respect to the dimensionless temporal variable $\tau$.

We make no assumption regarding the order of magnitude of the mass ratio; that is, at this point we do not restrict our analysis to small values of $\epsilon_{\mathrm{r}}$. Moreover, the stiffness of 


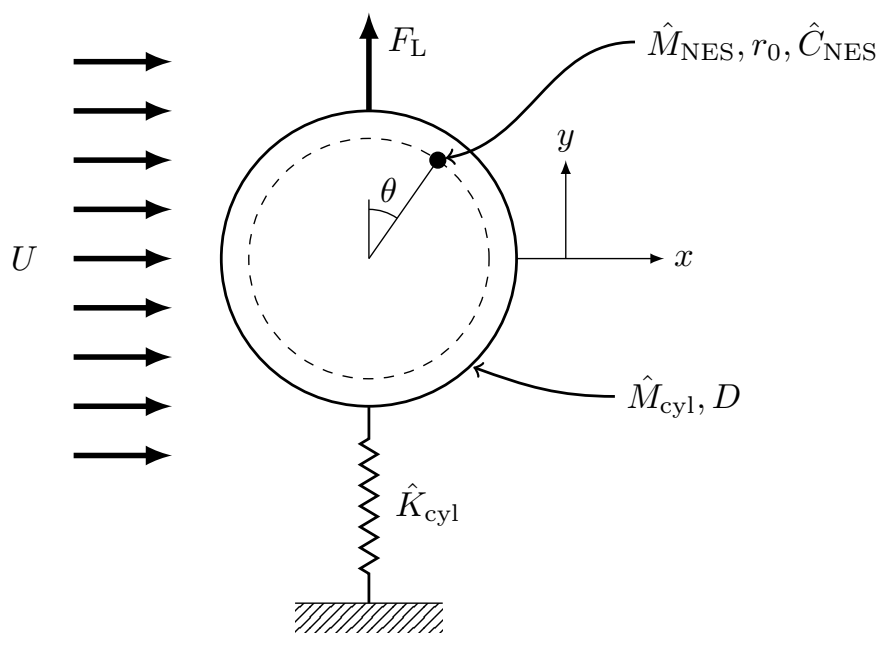

Figure 1: Schematic of the circular cylinder in cross-flow with rotational NES.

the spring supporting the cylinder is chosen so that the dimensionless natural frequency $f_{n}^{*}$ is close to the normalized natural shedding frequency of a stationary circular cylinder at $R e=100$, which is equal to the Strouhal number $S t=0.167$. Linearization of (11) about any of its equilibrium positions yields no linear terms, which makes the inertial coupling between the rotational NES and the primary structure (the cylinder) essentially nonlinear. With the addition of weak dissipation, characterized in the system by $\lambda_{\mathrm{r}}$, targeted energy transfer (TET) from the cylinder to the rotating mass is possible, as the essential nonlinearity confers the NES with the ability to engage in resonance capture with the cylinder dynamics and the surrounding flow over a broad range of frequencies (since it does not have a preferential linearized resonance frequency). Indeed, it has been shown that, under certain conditions [37], the resulting energy transfer from the cylinder and the surrounding flow to the rotator occurs in a one-way, nearly irreversible fashion, which makes the NES an efficient passive vibration-suppression device [8, 31].

In the present work, the fluid-structure interaction is studied computationally and analytically. Our computational approach is based on the spectral-element code Nek5000 $[11,14]$, having exponentially rapid convergence as the order of the polynomial basis functions increases. The Navier-Stokes equations are cast in an Arbitrary Eulerian Lagrangian (ALE) frame and integrated forward in time using an unconditionally-stable time-stepping scheme based on second-order backward-differentiation / extrapolation (BDF2/EXT2) discretization formulas. Equations $(11,12)$ governing the motions of the two coupled oscillators are solved using a fourth-order Runge-Kutta scheme with time-step size $\Delta \tau=2 \times 10^{-3}$.

The coupled system of equations $(11,12)$ and the discretized equations governing the motion of the fluid flow, used to evaluate the lift coefficient, are solved in a staggered fashion; i.e., we have assumed that, in the solution of the fluid-structure interaction problem, there is no need for equilibrating iterations in each time step, since the structure considered here is rigid, and provided that the time-step size is chosen small enough. This assumption was shown to be satisfactory by Tumkur [33], who performed computations 
in which the fluid and structural responses at each time step were computed iteratively, with insignificant effects on the response of the system (compared to the solution obtained without equilibrating iterations).

The circular cylinder is initially at rest, while the NES initial conditions are selected as $\theta(0)=\pi / 2$ and $\dot{\theta}(0)=0$. We mention that we observed no sensitivity of the phenomena reported thereafter to changes in the initial value of the NES angular displacement (with other initial conditions set to zero). In other cases (e.g., non-zero initial conditions for cylinder and rotator), however, strong sensitivity of the long-term solution to initial conditions is generally expected. In all simulations presented below, the density ratio $m^{*}$ is set equal to 10 , and the Reynolds number is fixed to $R e=100$. These two parameters are chosen to facilitate comparison of our numerical results with previous studies that have been carried out for VIV with and without an internal NES.

\subsection{Physical phenomena and motivation}

Recent works [33, 36] have established the existence of at least three passive VIVsuppression mechanisms that have been attributed to the interaction between the flow, the circular cylinder and the rotational NES. The first suppression mechanism reported by Tumkur et al. is characterized by a strongly modulated response of the cylinder which undergoes relaxation oscillations, and whose transverse displacement is reduced by about $71 \%$ compared to the NES-less system. The second suppression mechanism, which shows a reduction of nearly $50 \%$ of the cylinder displacement, is characterized by a 1:1:1 resonance lock-in between the NES, the cylinder and the lift force. In what follows, however, we focus on the third suppression mechanism, which features repetitive cycles of slowly decaying cylinder motion interrupted by intermittent chaotic oscillations [33, 36]. Indeed, the strongly modulated response of the first suppression mechanism is ubiquitous in systems comprising an NES under external forcing or self-excitation, while the second suppression mechanism is characterized by a periodic long-time solution. In contrast, the third suppression mechanism is by far the most interesting of the three, as it refers to neither periodic nor quasi-periodic long-time solutions, but rather exhibits significantly richer and more complex dynamics that will be described and investigated shortly. We also emphasize that the striking changes in the wake structure reported thereafter are specific to the third suppression mechanism, and that no comparable effects on the flow were found in the first two suppression mechanisms, for which the wake structure remains quite similar to that of the fixed-cylinder Kármán vortex street, with small differences being attributable to cylinder motion.

For NES parameters $\epsilon_{\mathrm{r}}=0.33, \lambda_{\mathrm{r}}=0.002745$ and $\bar{r}_{0}=0.458$, the fluid-structure interaction is characterized by successive cycles of regular motion of the cylinder and the NES interspersed by chaotic bursts. During periods of regular motion, the amplitude of the oscillations of the cylinder gradually decreases and the rotational NES becomes locked in a regime of steady rotation that corresponds to 1:1 resonance capture with the cylinder (i.e., the frequency of the NES rotation becomes nearly equal to the slowly decreasing frequency of vertical oscillation of the cylinder). Moreover, as the slowly decaying cycle progresses, the amplitude of the lift coefficient reduces drastically, and so does that of the drag coefficient, which assumes a nearly constant value toward the end of the cycle. Frequency analysis shows that, during the slowly decaying cycle, the cylinder, the NES and the lift force are clearly in 1:1:1 resonance. Hence, for this set of NES parameters, the responses of the three subsystems - the cylinder, the NES and the flow - are 
dominated by a single frequency close, but not equal, to the Strouhal frequency. This dominant frequency slowly decreases with time and reaches a minimum right before the slowly decaying cycle of the cylinder motion is destroyed, and the dynamics transitions toward instability, a phenomenon that has been correlated to the increase of NES-induced effective mass of the coupled system as the cycle progresses [36]. This phenomenon is demonstrated in the series of numerical simulations in Figs. 2(a, c, e) and 5(b, d, f, h).

Even more interesting, as the lift coefficient approaches zero and the drag coefficient tends to a local minimum, the structure of the flow aft of the cylinder changes noticeably. In the far field, the strength of the vortices is significantly lower compared to the fixed-cylinder Karman vortex street and the standard VIV case (linearly sprung cylinder without NES, see Fig. 3). In the near field, the attached vorticity is considerably elongated and straightened, which strongly suggests that the steady, symmetric solution unstable at that Reynolds number - is partially stabilized due to the action of the NES on the flow, mediated by the cylinder motion [36].

Motivated by these results, obtained for this particular set of NES parameters, we perform a parametric study to determine over what range of parameters the slowly decaying behavior exists, and how the time responses of the dynamical system as well as the structure of the wake are affected by changes in NES parameters. Time-frequency analysis is performed by means of the Morlet wavelet transform [1, 19], which provides insight into the dynamic evolutions of the main frequency components of the transient responses. In the wavelet transform contour plots shown below, dark and light areas correspond to regions where the amplitude of the wavelet transform is high and low, respectively. Such plots allow for identification of transitions between different regimes of the response as the nonlinear system evolves in time, and can thus be regarded as "dynamic analogs" of the classic Fourier transform, whose applicability is restricted to stationary signals and which provides the frequency content of a signal in an averaged sense $[27,37]$. Other techniques such as the Hilbert-Huang Transform $[24,26]$ that are widely used in reduced-order modeling of nonlinear systems have limited relevance to the present analysis and therefore will not be considered here.

An extensive series of numerical simulations indicated that this mechanism does not persist for values of the mass ratio exceeding 0.4. Therefore, for each value of $\epsilon_{\mathrm{r}}$ between 0.05 and 0.35 , we coarsely scan the parameter space to determine what (not necessarily unique) combinations of damping coefficient and rotator radius lead to the slowly decaying behavior. The results of this parametric search are compiled in Fig. 4, which shows combinations of NES parameters for which intermittent chaotic bursts followed by slowly decaying motion were observed.

There is no a priori reason that such region should be continuous in the parameter space, although both $\lambda_{\mathrm{r}}$ and $\bar{r}_{0}$ appear to depend continuously and monotonically on the mass ratio, rather than randomly. Therefore, Fig. 4 should not be interpreted as an indication that there exists a continuous region containing the type of previously described solutions, but merely as a demonstration that the slowly decaying cycle exists over a wide range of NES parameters. There may be a deterministic dependence of $\lambda_{\mathrm{r}}$ and $\bar{r}_{0}$ on $\epsilon_{\mathrm{r}}$; however, whether this observation bears any physical significance is questionable and lies beyond the scope of the present work.

Among all triplets of NES parameters represented in Fig. 4, we identify at least two distinct mechanisms by which the system alternates between slowly decaying cycles and instability bursts, as shown in Fig. 2. For values of $\epsilon_{\mathrm{r}}$ between 0.05 and 0.18 (cf. 

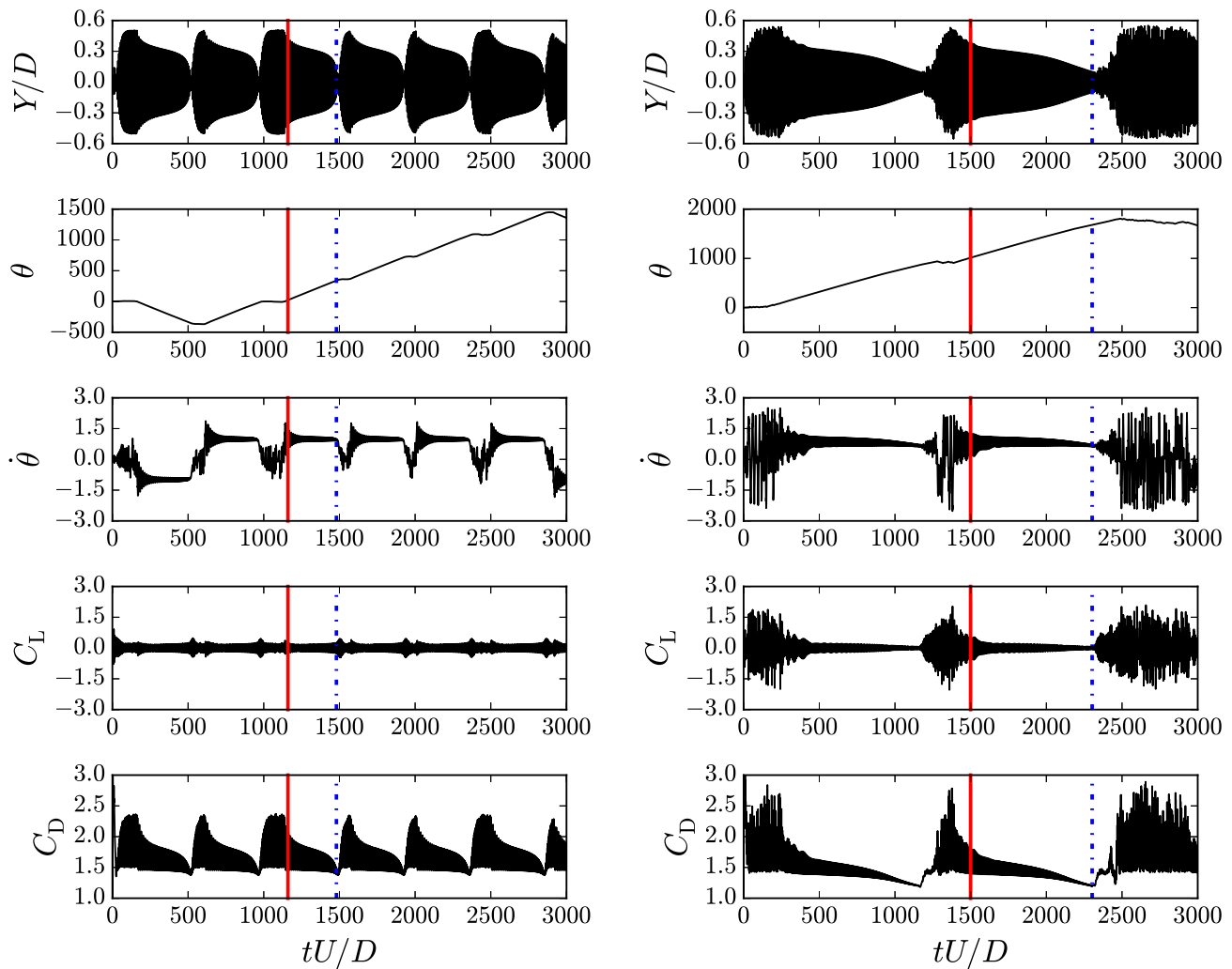

(a)

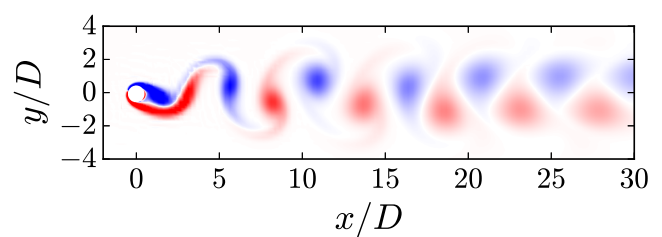

(b)

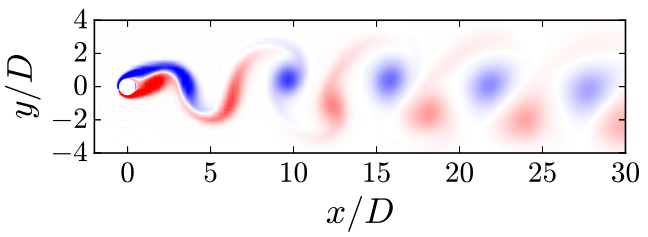

(c) Vorticity at $t U / D=1159.6$ identified by - in (a).

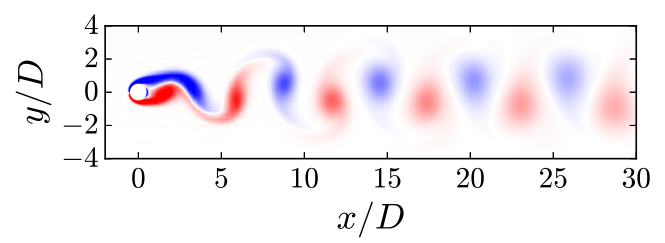

(d) Vorticity at $t U / D=1499.2$ identified by — in (b).

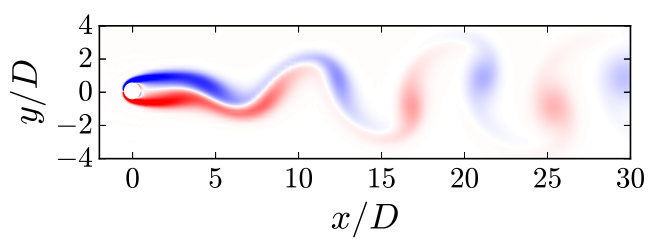

(f) Vorticity at $t U / D=2302.4$

identified by $-\cdot-$ in (b).

Figure 2: Time series and vorticity distributions at the beginning and end of the slowly decaying cycle for NES parameters $(\mathrm{a}, \mathrm{c}, \mathrm{e}) \epsilon_{\mathrm{r}}=0.05, \bar{r}_{0}=0.85$ and $\lambda_{\mathrm{r}}=0.054$; and $(\mathrm{b}, \mathrm{d}, \mathrm{f}) \epsilon_{\mathrm{r}}=0.33, \bar{r}_{0}=0.458$ and $\lambda_{\mathrm{r}}=0.002745$. 


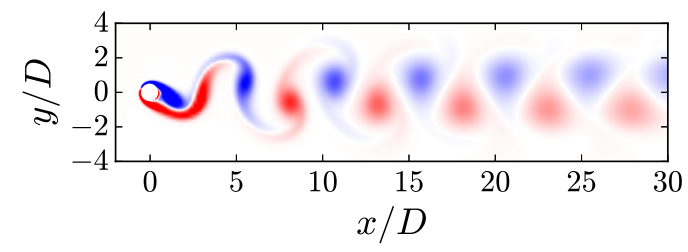

Figure 3: Spanwise vorticity field for standard VIV with no NES.

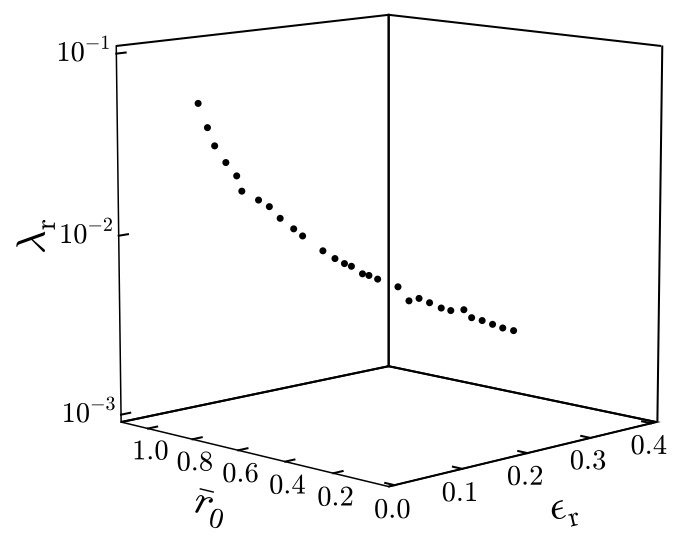

Figure 4: NES parameters for which repetitive cycles of slowly decaying oscillations of the cylinder with intermittent chaotic bursts leading to partial wake stabilization are observed. 
Fig. 2a) the response of the cylinder during the slowly decaying portion is dominated by a frequency that remains very close to the Strouhal number (see Fig. 5(a, c, e, g)) . Additional harmonics may be observed for values of $\epsilon_{\mathrm{r}}$ between 0.09 and 0.18 , owing to strongly nonlinear resonance interactions between the NES, the cylinder and fluid modes (these additional harmonics seem much less pronounced or even absent from the response for values of $\epsilon_{\mathrm{r}}$ smaller than 0.09). The decay in the amplitude of cylinder oscillations is significantly slower than in the reference case discussed earlier. The amplitude of the lift coefficient does not exhibit strong variations throughout the cycle. As will be investigated later, such small values of $\epsilon_{\mathrm{r}}$ (less than 0.1) are desirable in that they fall within the range of traditional asymptotic analysis, where the mass ratio is the small parameter of the problem.

For values of $\epsilon_{\mathrm{r}}$ between 0.23 and 0.4 ( $c f$. Fig. 2b), the response of the system remains qualitatively similar to what has been previously reported by Tumkur et al. [36]. That is, during the slowly decaying portion of the solution, the cylinder and NES responses are dominated by a single, slowly decreasing frequency with low-amplitude or even nonexistent higher or lower harmonics (see Fig. 5(b, d, f, h)). Moreover, the decays in amplitude of the cylinder, drag force and lift force oscillations are much more pronounced compared to lower values of $\epsilon_{\mathrm{r}}$. The minimum value of the drag force reached toward the end of the slowly decaying cycle diminishes as well. The drastic change in the vorticity field in the wake of the cylinder from the beginning to the end of the slowly decaying cycle is evident from Figs. 2d and 2f, as compared with Figs. 2c and 2e.

While it is clear that these two types of slowly decaying solutions are qualitatively different, we observe a "smooth" transition of the response as the value of the mass ratio is increased (and other NES parameters varied based on the empirical results shown in Fig. 4). In all cases, we surmise that the slowly decaying cycles interrupted by periods of chaotic motions are the result of a resonance capture on a slow invariant manifold of the fluid-structure-interaction dynamics. In what follows, we employ asymptotic analysis and scale-partitioning techniques to analytically investigate the existence and structure of the surmised invariant manifold, and thus identify the nonlinear dynamical mechanism that governs the partial stabilization of the wake aft of the cylinder during the slowly decaying portion of the dynamics, owing to the action of the internal nonlinear rotator.

\section{Order reduction of the fluid-structure interaction dynamics}

We wish to analytically study the dynamics of the system governed by $(11,12)$ in the regime of resonance during which the slowly decaying cycle arises. As noted earlier, there are qualitative differences in the fluid-structure interaction dynamics depending on the value of the mass ratio $\epsilon_{\mathrm{r}}$. In particular, the slowly decaying cycle for low values of $\epsilon_{\mathrm{r}}$ features qualitatively different dynamics compared to finite values of $\epsilon_{\mathrm{r}}$. From an asymptotic perspective as well, these two cases significantly differ. It follows that the analytical treatment of the equations that ultimately reduces the dynamics to a loworder model needs to account for the size of parameter $\epsilon_{\mathrm{r}}$ compared to the other system parameters involved in the problem, which leads us to consider two cases, namely small and finite values of the mass ratio.

A critical aspect of the reduced-order analysis is the treatment of the lift coefficient, which is the only term in (11) that couples the motion of the cylinder (and indirectly of the NES) to that of the fluid, and for which an explicit expression as a function 


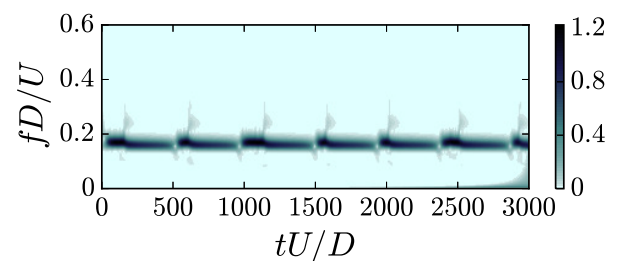

(a) Cylinder displacement $Y / D$

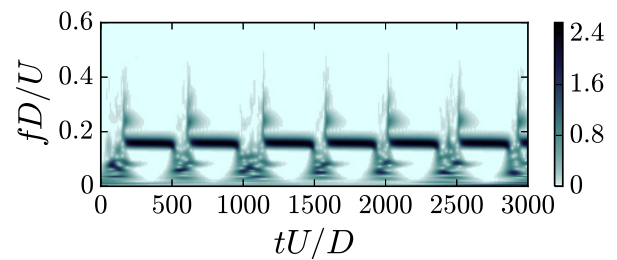

(c) Sine of angular displacement $\sin \theta$

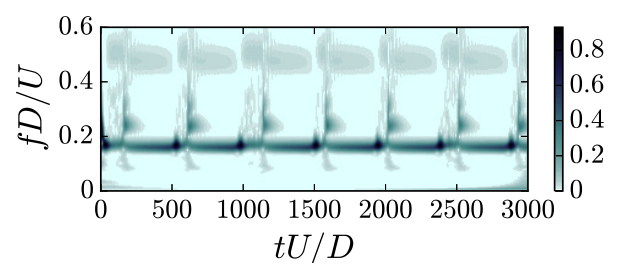

(e) Lift coefficient $C_{\mathrm{L}}$

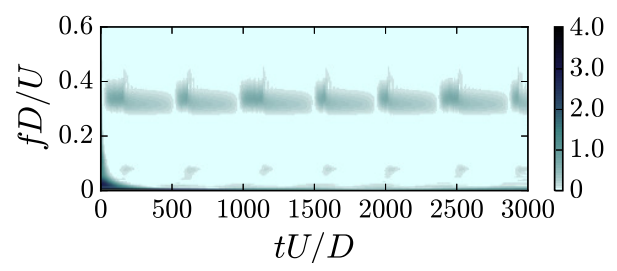

(g) Drag coefficient $C_{\mathrm{D}}$

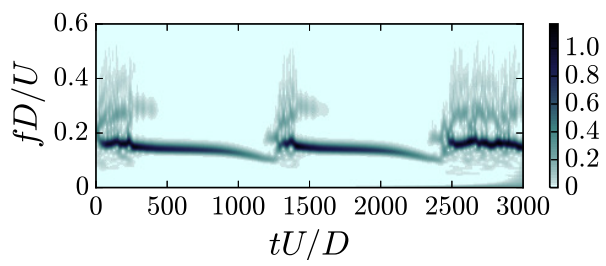

(b) Cylinder displacement $Y / D$

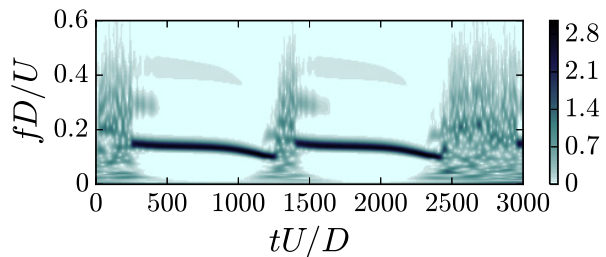

(d) Sine of angular displacement $\sin \theta$

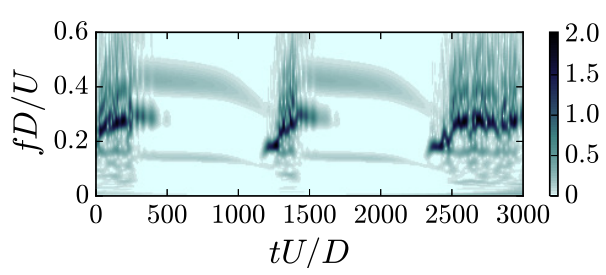

(f) Lift coefficient $C_{\mathrm{L}}$

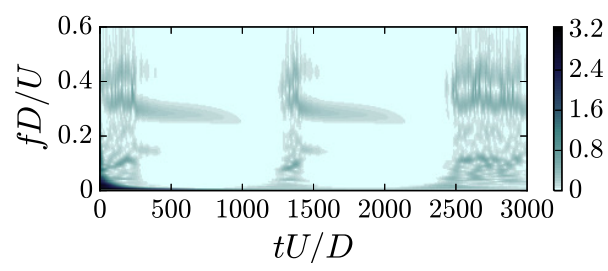

(h) Drag coefficient $C_{\mathrm{D}}$

Figure 5: Wavelet spectra of component dynamics for NES parameters (a, c, e, g) $\epsilon_{\mathrm{r}}=0.05, \bar{r}_{0}=0.85$ and $\lambda_{\mathrm{r}}=0.054 ;$ and $(\mathrm{b}, \mathrm{d}, \mathrm{f}, \mathrm{h}) \epsilon_{\mathrm{r}}=0.33, \bar{r}_{0}=0.458$ and $\lambda_{\mathrm{r}}=0.002745$. 
of time is not available since it it determined by numerically solving the Navier-Stokes equations governing the fluid motion. There are at least two options to construct an approximate model of the temporal evolution of the lift coefficient. One approach consists in considering an "open-loop" interaction between the fluid and the cylinder, in which the lift coefficient is simply replaced with an a priori known, prescribed forcing term on the right-hand side of (11). Numerical data of the dimensionless lift force $C_{\mathrm{L}}$ during the regime of interest generally provides enough information to formulate such ansatz, which for our purposes can be taken as a simple single-frequency harmonic forcing. Other, more elaborate reduced-order models of the fluid-structure interaction dynamics entail the use of a fluid oscillator, i.e., the addition to the original 2DOF system $(11,12)$ of (generally) one ordinary differential equation that governs the evolution of the lift coefficient and couples it to that of the cylinder. Van-der-Pol-type and Rayleigh-type oscillators are typically good candidates for such "closed-loop" modeling [13, 15, 23, 25, 29].

In the following, we opt for the first approach and use a data-driven modeling procedure of the lift coefficient, which we consider as an external forcing term on the right-hand side of (11). The form of the approximate ansatz $\hat{C}_{\mathrm{L}}$ is dictated by conclusions drawn from time histories and wavelet spectra (see Figs. 2 and 5) obtained by direct numerical simulations. As will be shown, this approach yields excellent results, despite its deceiving simplicity.

\subsection{Case of small mass ratios}

When the mass ratio is small (i.e., $\epsilon_{\mathrm{r}} \ll 1$ ), it may be considered as the small parameter of the asymptotic analysis. Therefore, in this section, we drop the subscript in $\epsilon_{\mathrm{r}}$ for convenience. The time series obtained from direct numerical simulation of the fluid-structure interaction using Nek5000 show that, in this particular case, the dominant frequency of the lift coefficient does not deviate much from the Strouhal frequency (see also Figs. 5(a, c, e, g)). Further, the amplitude of the lift coefficient is fairly constant throughout the slowly decaying cycle, and roughly of the same order of magnitude as that of the mass ratio. Therefore, in the limit of small mass ratios, the lift coefficient is approximated by the ansatz

$$
\frac{2 \hat{C}_{\mathrm{L}}}{\pi m^{*}}=\epsilon A \sin (\omega \tau+\eta) .
$$

In this expression, $A$ is an $\mathcal{O}(1)$ quantity, $\eta$ accounts for possible phase lag with the cylinder oscillations, and $\omega=\omega_{\mathrm{r}}^{*}(1+\epsilon \sigma)$, where $\sigma$ characterizes the small detuning from the Strouhal frequency. Under these assumptions, we rewrite the normalized equations for the cylinder and the rotational NES $(11,12)$ as

$$
\begin{aligned}
\ddot{y}+\epsilon \lambda_{\mathrm{s}} \dot{y}+\omega_{\mathrm{r}}^{* 2} y & =\epsilon \bar{r}_{0} \frac{\mathrm{d}}{\mathrm{d} \tau}(\dot{\theta} \sin \theta)+\epsilon A \sin (\omega \tau+\eta) \\
\ddot{\theta}+\lambda_{\mathrm{r}} \dot{\theta} & =-\omega_{\mathrm{r}}^{* 2} \frac{y}{\bar{r}_{0}} \sin \theta+\mathcal{O}(\epsilon),
\end{aligned}
$$

where we have included an additional term, $\epsilon \lambda_{\mathrm{s}} \dot{y}$, that accounts for weak linear viscous damping of the cylinder motion and makes the analysis more general, although the damping coefficient $\lambda_{\mathrm{s}}$ was set to zero in all numerical simulations. 
In the next step, we perform an asymptotic analysis of the system $(14,15)$ based on a slow/fast partition of the transient dynamics in the vicinity of the regime of 1:1:1 resonance by applying the method of complexification-averaging (CX-A) as described by Manevitch [28]. To this end, we introduce the representations

$$
\begin{aligned}
\dot{y}(\tau)+\mathrm{j} \omega y(\tau) & =\phi(\tau) \mathrm{e}^{\mathrm{j} \omega \tau} \\
\theta(\tau) & = \pm \omega \tau+\psi(\tau),
\end{aligned}
$$

and, assuming time-scale separation, we partition the dynamics of the system in terms of two independent slow and fast scales. Here, $\phi$ is the complex amplitude that characterizes modulation of the fast cylinder oscillations, and $\psi$ the real, slowly varying phase modulation of the NES rotation, both of which vary on a time scale slower than the "fast" scale $\omega \tau$. The plus-or-minus sign in (17) accounts for the direction of revolving motion of the rotational NES during the slowly decaying cycle, which can be clockwise $(+)$ or counterclockwise $(-)$. We emphasize that one additional assumption is made here, namely that the NES and cylinder transient responses as well as the lift coefficient are dominated by a single, fast frequency $\omega$, and that all other harmonics observed in the wavelet spectra are in comparison negligible. Note that such assumptions may not hold in other regimes of the fluid-structure-interaction dynamics, in which additional "fast" frequencies may appear and need to be accounted for in the analysis; however, they are consistent with the numerical simulations depicted in Figs. 2 and 5 for small mass ratio $\left(\epsilon_{\mathrm{r}}=0.1\right)$. Examples of applications of the CX-A method in the broader context of multi-frequency dynamics can be found in [37].

Assuming that the NES rotates clockwise, we substitute $(16,17)$ in $(14,15)$ and average with respect to the "fast" scale to obtain the set of two complex modulation equations

$$
\begin{aligned}
\dot{\phi}+\epsilon\left(\mathrm{j} \omega_{\mathrm{r}}^{*} \sigma+\frac{\lambda_{\mathrm{s}}}{2}\right) \phi & =\frac{\epsilon \bar{r}_{0}}{2}\left[\left(\omega_{\mathrm{r}}^{*}+\dot{\psi}\right)^{2}-\mathrm{j} \ddot{\psi}\right] \mathrm{e}^{\mathrm{j} \psi}+\frac{\epsilon A}{2 \mathrm{j}} \mathrm{e}^{\mathrm{j} \eta}+\mathcal{O}\left(\epsilon^{2}\right) \\
\ddot{\psi}+\lambda_{\mathrm{r}}\left(\omega_{\mathrm{r}}^{*}+\dot{\psi}\right) & =-\frac{\omega_{\mathrm{r}}^{*}}{4 \bar{r}_{0}}\left(\phi \mathrm{e}^{-\mathrm{j} \psi}+\tilde{\phi} \mathrm{e}^{\mathrm{j} \psi}\right)+\mathcal{O}(\epsilon),
\end{aligned}
$$

where () denotes complex conjugation. This set represents the slow flow of the fluidstructure interaction in the 1:1:1 resonance regime. It should be clear that these equations are valid only under the assumptions described above as they do not contain any harmonic terms with frequencies higher than the basic fast frequency $\omega$.

The slow-flow equations are further reduced by applying the method of multiple scales. We introduce new independent time scales $\tau_{k}=\epsilon^{k} \tau, k \in \mathbb{N}$, such that the derivative with respect to the slow time scale $\tau$ can be expanded as

$$
\frac{\mathrm{d}()}{\mathrm{d} \tau}=\frac{\partial()}{\partial \tau_{0}}+\epsilon \frac{\partial()}{\partial \tau_{1}}+\cdots
$$

We note that whereas $\tau_{0}=\tau$ is the original slow time scale of the problem, the higherorder time scales $\tau_{1}, \tau_{2}$, etc, represent super-slow time scales of the asymptotic analysis. Similarly, we assume that the solution of $(18,19)$ can be expanded in the form

$$
\begin{aligned}
& \phi=\phi_{0}\left(\tau_{0}, \tau_{1}, \cdots\right)+\epsilon \phi_{1}\left(\tau_{0}, \tau_{1}, \cdots\right)+\cdots \\
& \psi=\psi_{0}\left(\tau_{0}, \tau_{1}, \cdots\right)+\epsilon \psi_{1}\left(\tau_{0}, \tau_{1}, \cdots\right)+\cdots .
\end{aligned}
$$


Substituting $(20)$ to $(22)$ in $(18,19)$, and collecting coefficients involving the same powers of $\epsilon$ leads to a hierarchy of subproblems governing the leading-order approximations of the solution to the slow flow equations.

The $\mathcal{O}(1)$ subproblem is given by

$$
\frac{\partial \phi_{0}}{\partial \tau_{0}}=0 \Longrightarrow \phi_{0}=\phi_{0}\left(\tau_{1}, \cdots\right) .
$$

With (23) in hand, we introduce the polar transformation

$$
\phi_{0}=N\left(\tau_{1}\right) \mathrm{e}^{\mathrm{j} \delta\left(\tau_{1}\right)},
$$

where $N$ and $\delta$ are real, slowly varying, leading-order amplitude and phase of the cylinder oscillation, respectively; and rewrite (19) as

$$
\frac{\partial^{2} \psi_{0}}{\partial \tau_{0}{ }^{2}}+\lambda_{\mathrm{r}}\left(\omega_{\mathrm{r}}^{*}+\frac{\partial \psi_{0}}{\partial \tau_{0}}\right)=-\frac{\omega_{\mathrm{r}}^{*}}{2 \bar{r}_{0}} N \cos \left(\psi_{0}-\delta\right) .
$$

Note that $N$ and $\delta$ are functions of $\tau_{1}$ by virtue of (23), and therefore are treated as constants in (25), which is governed by the time scale $\tau_{0}$. The latter is nothing else but the equation governing the motion of a pendulum subject to a constant torque and restrained by linear viscous damping. The response of this system, depending on the initial conditions and parameter values, can be attracted either to a limit cycle or to one of two fixed points of opposite stability. While limit cycle oscillations always exist, the pair of stable/unstable fixed points exist only for certain parameter values [22]. Quite clearly, the fixed points of (25) correspond to stable rotations characterizing the state of exact 1:1:1 resonance that is of main interest in this study, and must thereby satisfy

$$
-2 \lambda_{\mathrm{r}} \bar{r}_{0}=N \cos (\delta-\gamma), \quad \gamma=\lim _{\tau_{0} \rightarrow \infty} \psi_{0}\left(\tau_{0}, \tau_{1}, \cdots\right),
$$

which has solutions if and only if

$$
2 \lambda_{\mathrm{r}} \bar{r}_{0} \leq N\left(\tau_{1}\right) .
$$

Equation (26) defines the slow invariant manifold (SIM) of the problem. Considering its topological features, it is composed of two branches corresponding to each possible solution of (26), and breaks down by the mechanism of saddle-node bifurcation when (27) becomes an equality. Past this point, the slow flow leaves the regime of 1:1:1 resonance and enters a different regime of dynamics. A quick linear stability analysis shows that the stable branch must satisfy $\sin (\delta-\gamma)>0$, as depicted in Fig. 6. Also, note that the topological structure of the SIM depends on the direction of rotation of the pendulum rotator since, when the NES rotates counter-clockwise (i.e., $\theta=-\omega \tau+\psi)$, the SIM is defined by $-2 \lambda_{\mathrm{r}} \bar{r}_{0}=N \cos (\delta+\gamma)$.

We proceed to investigate the dynamics of the fluid-structure interaction at the superslow scale on the stable branch of the SIM. To this end, we recognize that in the vicinity of the SIM all derivatives of the phase $\psi_{0}$ can be neglected (by the very definition of the SIM). Collecting terms of $\mathcal{O}(\epsilon)$ in (18), and arguing that secular terms in $\tau_{0}$ should vanish, we obtain the complex super-slow modulation equation

$$
\frac{\partial \phi_{0}}{\partial \tau_{1}}+\left(\mathrm{j} \omega_{\mathrm{r}}^{*} \sigma+\frac{\lambda_{\mathrm{s}}}{2}\right) \underset{14}{\phi_{0}}=\frac{\omega_{\mathrm{r}}^{* 2} \bar{r}_{0}}{2} \mathrm{e}^{\mathrm{j} \gamma}+\frac{A}{2 \mathrm{j}} \mathrm{e}^{\mathrm{j} \eta},
$$




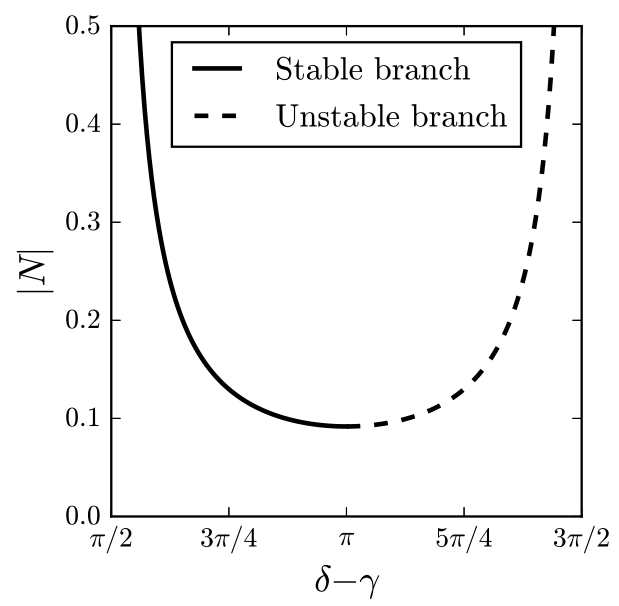

Figure 6: Slow invariant manifold (SIM) for $\lambda_{\mathrm{r}}=0.054$ and $\bar{r}_{0}=0.85$.

which we cast into two real equations using (24),

$$
\begin{aligned}
\frac{\partial N}{\partial \tau_{1}}+\frac{\lambda_{\mathrm{s}}}{2} N & =\frac{\omega_{\mathrm{r}}^{* 2} \bar{r}_{0}}{2} \cos (\gamma-\delta)+\frac{A}{2} \sin (\eta-\delta) \\
N \frac{\partial \delta}{\partial \tau_{1}}+\omega_{\mathrm{r}}^{*} \sigma N & =\frac{\omega_{\mathrm{r}}^{* 2} \bar{r}_{0}}{2} \sin (\gamma-\delta)-\frac{A}{2} \cos (\eta-\delta) .
\end{aligned}
$$

These slow modulation equations (the super-slow flow of the dynamics) govern the superslow evolution of the amplitude and phase of the response on the stable branch of the SIM, respectively. Equations $(29,30)$ are in general not solvable analytically, except in the unforced case when $A=0$, as shown by Sigalov et al. [31].

We look for the fixed points of the super-slow flow by setting all the derivatives to zero in $(29,30)$ and recognizing that, on the stable branch of the SIM, $-2 \lambda_{\mathrm{r}} \bar{r}_{0}=N \cos (\delta-\gamma)$ and $\sin (\delta-\gamma)>0$. This observation leads to

$$
\begin{aligned}
\frac{\lambda_{\mathrm{s}}}{2} N & =-\frac{\omega_{\mathrm{r}}^{* 2} \bar{r}_{0}^{2} \lambda_{\mathrm{r}}}{N}+\frac{A}{2} \sin (\eta-\delta) \\
\omega_{\mathrm{r}}^{*} \sigma N & =-\frac{\omega_{\mathrm{r}}^{* 2} \bar{r}_{0}}{2} \sqrt{1-\frac{4 \bar{r}_{0}^{2} \lambda_{\mathrm{r}}}{N^{2}}}-\frac{A}{2} \cos (\eta-\delta) .
\end{aligned}
$$

We then eliminate those terms that involve the phase difference $\eta-\delta$ and arrive to the conclusion that the fixed points of the super-slow flow, if any, must satisfy the expression

$$
\left(\frac{\lambda_{\mathrm{s}}}{2} N+\frac{\omega_{\mathrm{r}}^{* 2} \bar{r}_{0}^{2} \lambda_{\mathrm{r}}}{N}\right)^{2}+\left(\omega_{\mathrm{r}}^{*} \sigma N+\frac{\omega_{\mathrm{r}}^{* 2} \bar{r}_{0}}{2} \sqrt{1-\frac{4 \bar{r}_{0}^{2} \lambda_{\mathrm{r}}}{N^{2}}}\right)^{2}=\frac{A^{2}}{4} .
$$

It appears that (33) admits real solutions only if the amplitude $A$ of the surrogate lift force is large enough, which is consistent with the physics of the problem since insufficient 
forcing cannot counterbalance dissipation owing to the viscosity of the surrounding fluid and to the dissipative internal rotator (the NES). On the other hand, one expects that, if the value of $A$ is large enough, at least one stable fixed point should appear. A similar feature of the SIM has been observed for a vibro-impact NES attached to a linear oscillator subject to harmonic forcing, in which case numerical simulations show that the existence of fixed points on the stable branch of the SIM highly depends on the magnitude of the external forcing [17].

We note that, in the present system, the smallest value that the forcing amplitude $A$ should take in order for a fixed point to exist on the SIM is computed by letting $N=2 \lambda_{\mathrm{r}} \bar{r}_{0}$ in (33), that is,

$$
A=\bar{r}_{0} \sqrt{\left(2 \lambda_{\mathrm{s}} \lambda_{\mathrm{r}}+\omega_{\mathrm{r}}^{* 2}\right)^{2}+\left(4 \omega_{\mathrm{r}}^{*} \lambda_{\mathrm{r}} \sigma\right)^{2}},
$$

which represents the "ideal" scenario (from the viewpoint of vibration suppression) in which the fixed point is located at $N=2 \lambda_{\mathrm{r}} \bar{r}_{0}$. Indeed, this particular point corresponds to the minimum amplitude of cylinder motion $N$ that can be reached on the stable branch of the SIM, due to its bowl-like topological structure shown in Fig. 6. In the present FSI problem, however, the magnitude of the external forcing $A$ is not prescribed, but rather results from the interaction between the fluid flow and the freely oscillating cylinder. For NES parameters used in Fig. 2(a, c, e), the amplitude of the lift force should a minima reach $C_{\mathrm{L}}=0.755$ for a fixed point to appear, which is clearly never realized, as evidenced by the time series.

\subsection{Case of finite mass ratios}

For large values of mass ratio $\left(\epsilon_{\mathrm{r}}>0.1\right)$, the above asymptotic analysis loses its validity, for two main reasons. First, the mass ratio cannot be considered as the small parameter of the problem anymore and it becomes impossible to apply the method of multiple scales as was done before. Second, the numerical simulations clearly show that, in this case, the instantaneous frequency $\omega$ deviates continuously and significantly from the Strouhal frequency as the slowly decaying cycle progresses. Likewise, the amplitude of the lift coefficient slowly decreases with time, which renders (13) unusable. Therefore, we express the equations of motion as

$$
\begin{aligned}
\ddot{y}+\lambda_{\mathrm{s}} \dot{y}+\omega_{\mathrm{r}}^{* 2} y & =\epsilon_{\mathrm{r}} \bar{r}_{0} \frac{\mathrm{d}}{\mathrm{d} \tau}(\dot{\theta} \sin \theta)+\frac{2 \hat{C}_{\mathrm{L}}}{\pi m^{*}} \\
\ddot{\theta}+\lambda_{\mathrm{r}} \dot{\theta} & =\frac{\ddot{y}}{\bar{r}_{0}} \sin \theta,
\end{aligned}
$$

where, here again, we have included a structural damping term that is not present in the numerical simulations. In order to apply complexification-averaging techniques, we impose the condition of 1:1:1 resonance and introduce the instantaneous frequency $\omega=$ $\omega(\tau)$ that depends on the amplitude of oscillations of the cylinder. Such approach was first successfully used by Domany and Gendelman [12] who analytically investigated the dynamics of a Van der Pol-Duffing oscillator with NES for which large frequency variations may be observed in the response. Then, we introduce the change of variables

$$
\begin{aligned}
R(\tau) \mathrm{e}^{\mathrm{j} \zeta(\tau)} & =\dot{y}(\tau)+\mathrm{j} \omega(\tau) y(\tau) \\
\theta(\tau) & = \pm \zeta(\tau)+\psi(\tau),
\end{aligned}
$$


where a slow-fast partition of the dynamics is used, with $R(\tau)$ and $\zeta(\tau)$ being the (real) slowly varying amplitude and fast varying phase of the cylinder response, respectively, and $\psi(\tau)$ being the slowly varying correction to the phase of the NES rotation. The instantaneous frequency $\omega$ and "fast" phase $\zeta$ over which the equations of motion will be averaged are related through the expression

$$
\dot{\zeta}(\tau)=\omega(\tau) .
$$

We also need to specify a formula for the ansatz lift force, which, in this case, we take to be

$$
\frac{2 \hat{C}_{\mathrm{L}}}{\pi m^{*}}=A(\tau) \sin [\zeta(\tau)+\eta(\tau)],
$$

where $A$ and $\eta$ are a priori assumed slowly varying functions of time. Equations (37) to (40) are consistent with the numerical results shown in Fig. 5(b, d, f, h) in that they incorporate the essential assumption that, in the regime of 1:1:1 resonance, the NES, the cylinder and the lift coefficient are dominated by a single, slowly varying frequency $\omega$ and, consequently, that additional small-amplitude harmonics may be neglected.

Substituting $(37)$ to $(40)$ in $(35,36)$ and averaging over the fast phase $\zeta$ yields the complex slow-flow equations

$$
\begin{gathered}
\dot{R}+\frac{R}{2}\left(\mathrm{j} \omega-\mathrm{j} \frac{\omega_{r}^{* 2}}{\omega}-\frac{\dot{\omega}}{\omega}+\lambda_{\mathrm{s}}\right)=-\frac{\epsilon_{\mathrm{r}} \bar{r}_{0}}{2}\left[\mathrm{j}(\dot{\omega}+\ddot{\psi})-(\omega+\dot{\psi})^{2}\right] \mathrm{e}^{\mathrm{j} \psi}+\frac{A}{2 \mathrm{j}} \mathrm{e}^{\mathrm{j} \eta} \\
\ddot{\psi}+\dot{\omega}+\lambda_{\mathrm{r}}(\omega+\dot{\psi})=\frac{\mathrm{j}}{2 \bar{r}_{0}}\left[(\dot{R}+\mathrm{j} \omega R) \mathrm{e}^{-\mathrm{j} \psi}-\left(\frac{\dot{\omega}}{\omega} R \cos \psi+\omega R \sin \psi\right)\right],
\end{gathered}
$$

derived under the assumption that the NES rotates clockwise. A similar expression, however, can be found for the case of counter-clockwise NES rotation. As a next step, we isolate real and imaginary parts in (42), as

$$
\begin{array}{ll}
\operatorname{Re}(\cdot): & \ddot{\psi}+\dot{\omega}+\lambda_{\mathrm{r}}(\omega+\dot{\psi})=\frac{1}{2 \bar{r}_{0}}(\dot{R} \sin \psi-\omega R \cos \psi) \\
\operatorname{Im}(\cdot): \quad \dot{R} \cos \psi+\omega R \sin \psi=\frac{\dot{\omega}}{\omega} R \cos \psi+\omega R \sin \psi
\end{array}
$$

Similarly to the case of small mass ratios examined in the previous section, the fixed points of these equations correspond to the state of 1:1:1 resonance that is of interest here. Setting all the derivatives to zero in (44) leads to a tautology, while a similar procedure applied to (43) yields

$$
R \cos \psi=-2 \lambda_{\mathrm{r}} \bar{r}_{0} .
$$

This relation defines the slow invariant manifold (SIM) of the fluid-structure interaction dynamics for finite mass ratios, with a slowly varying frequency in the dynamics. It is interesting to see that the topological structure of the SIM in this case is identical to that derived in (26) in the limit of small mass ratios. In particular, the SIM has two branches, the one stable, the other unstable. The difference, however, lies in the fact that there is an implicit dependence of the quantities involved in (45) on the instantaneous frequency $\omega$, a feature that was not observed in the case of the small mass ratio examined in the previous section. 


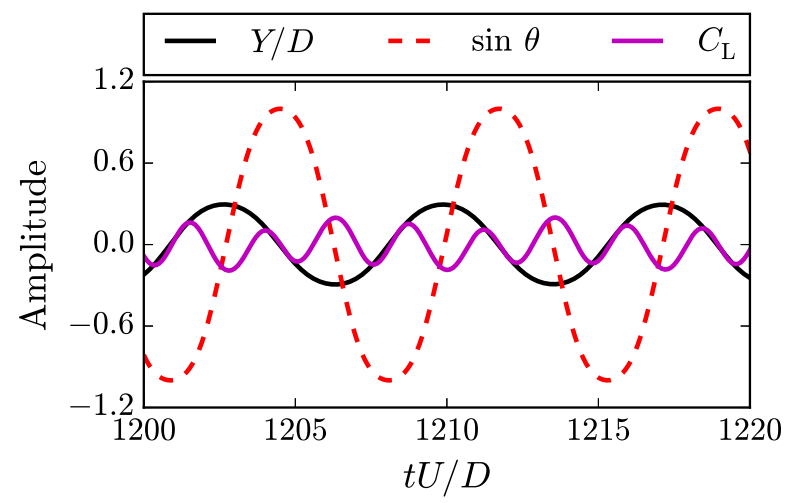

Figure 7: Time series of cylinder displacement, pendulum angular displacement and lift force during part of the slowly decaying cycle for NES parameters $\epsilon_{\mathrm{r}}=0.33, \bar{r}_{0}=0.458$ and $\lambda_{\mathrm{r}}=0.002745$.

The parametric study presented in section 2 has made it clear that, for larger values of $\epsilon_{\mathrm{r}}$, the value of the rotational damping coefficient must be very small for the slowly decaying cycle to occur (see Fig. 4). Moreover, the orders of magnitude of $R$ and $\bar{r}_{0}$ are comparable, which means that $\cos \psi$ must be of the same ("small") order of magnitude as $\lambda_{\mathrm{r}}$ for (45) to be consistent. Therefore, we expect to find that $\psi \approx \pm \pi / 2$, and decide on the sign of the phase lag by noting that, on the stable branch of the SIM, it must hold that $\sin \psi<0$ (which results from a linear stability analysis on the variational form of (43), similar to the case of small mass ratio). Comparison of the time series of cylinder displacement and sine of the NES angular displacement immediately validates this approximation, as shown in Fig. 7. From this graph, we also realize that the first harmonic of the lift coefficient oscillates in an out-of-phase fashion with respect to the cylinder, and therefore identify the slow phase $\eta$ as

$$
\eta(\tau)=\pi+\beta(\tau), \quad \beta(\tau) \ll 1 .
$$

The ansatz for the lift coefficient introduced in (40) is then slightly simplified as

$$
\frac{2 \hat{C}_{\mathrm{L}}}{\pi m^{*}}=-A(\sin \zeta+\beta \cos \zeta),
$$

where $A, \zeta$ and $\beta$ are assumed to be slowly varying with time.

At this points in analysis, we have exhausted all the information that (44) can provide, i.e., we have derived the expression of the slow invariant manifold in the regime of 1:1:1 resonance. We now turn to (41) and take the analysis to the super-slow scale. Arguing once again that, in the vicinity of the SIM, all derivatives of $\psi$ can be neglected, we extract from (41) real and imaginary parts, yielding

$$
\begin{array}{ll}
\operatorname{Re}(\cdot): & \dot{R}+\frac{R}{2}\left(\lambda_{\mathrm{s}}-\frac{\dot{\omega}}{\omega}\right)=\frac{\epsilon_{\mathrm{r}} \bar{r}_{0}}{2}\left(\dot{\omega} \sin \psi+\omega^{2} \cos \psi\right)-\frac{\beta A}{2} \\
\operatorname{Im}(\cdot): & \omega R-\frac{\omega_{\mathrm{r}}^{* 2}}{\omega} R=\epsilon_{\mathrm{r}} \bar{r}_{0}\left(-\dot{\omega} \cos \psi+\omega^{2} \sin \psi\right)+A .
\end{array}
$$


Further simplifications are made by invoking that, on the stable branch of the SIM, it holds that $\sin \psi \approx-1$ and $\cos \psi=-2 \lambda_{\mathrm{r}} \bar{r}_{0} / R$, which when substituted in the above equations yields the set of slow flow equations

$$
\begin{aligned}
\dot{R}+\frac{R}{2}\left(\lambda_{\mathrm{s}}-\frac{\dot{\omega}}{\omega}\right) & =-\frac{\epsilon_{\mathrm{r}} \bar{r}_{0}}{2}\left(\dot{\omega}+2 \lambda_{\mathrm{r}} \bar{r}_{0} \frac{\omega^{2}}{R}\right)-\frac{\beta A}{2} \\
\left(\omega^{2}-\omega_{\mathrm{r}}^{* 2}\right) R & =-\epsilon_{\mathrm{r}} \bar{r}_{0} \omega^{3}+\omega A .
\end{aligned}
$$

Note that, in the new slow flow equations $(50,51)$, we have neglected the term $\dot{\omega} \cos \psi$ as a product of two "small" quantities. Equation (51) can be regarded as a frequencyamplitude expression, relating the frequency of the cylinder oscillation to its amplitude. It is a cubic equation in $\omega$ and, given the set of NES parameters considered, it admits only one real root (and two complex conjugate roots), for which a closed-form expression can be derived as

$$
\omega(R)=-\frac{1}{3 \epsilon_{\mathrm{r}} \bar{r}_{0}}\left(R+C \mathrm{e}^{2 \mathrm{j} \pi / 3}+\frac{\Delta_{0}}{C} \mathrm{e}^{-2 \mathrm{j} \pi / 3}\right),
$$

where we have defined

$$
\begin{aligned}
\Delta_{0} & =R^{2}+3 \epsilon_{\mathrm{r}} \bar{r}_{0} A \\
\Delta_{1} & =2 R^{3}+9 \epsilon_{\mathrm{r}} \bar{r}_{0} R A-27 R\left(\epsilon_{\mathrm{r}} \bar{r}_{0} \omega_{\mathrm{r}}^{*}\right)^{2}, \\
C & =\sqrt[3]{\frac{\Delta_{1}+\sqrt{\Delta_{1}^{2}-4 \Delta_{0}^{3}}}{2}}
\end{aligned}
$$

Equation (52) explicitly relates the instantaneous frequency of the cylinder response $\omega$ to its amplitude $R$. Note that it involves the amplitude of external forcing $A$, which makes it intrinsically energy-dependent (which is a reflection of the nonlinearity of the problem). Differentiating (51) yields an expression for $\dot{\omega}$,

$$
\dot{\omega}=\frac{\dot{R}\left(\omega_{\mathrm{r}}^{* 2}-\omega^{2}\right)+\dot{A} \omega}{2 \omega R+3 \omega^{2} \epsilon_{\mathrm{r}} \bar{r}_{0}+A} .
$$

We recall at this point that, during the regime of 1:1:1 resonance, the amplitude of oscillations of the cylinder slowly decreases, and so does that of the lift coefficient. Therefore, it must hold that $\dot{R}<0$ and $\dot{A}<0$. In addition, wavelet spectra show that in this regime the instantaneous frequency $\omega$ deviates from the dimensionless natural frequency of the spring $\omega_{\mathrm{r}}^{*}$ in such a way that $\omega_{\mathrm{r}}^{* 2}-\omega^{2}>0$. These observations applied to (56) lead to the conclusion that $\dot{\omega}<0$, which is consistent with the slow decrease of the instantaneous frequency identified from the numerical results.

Equation (56) may then be substituted in (50) to obtain the final expression of the slow-flow equation

$$
\dot{R}=-\frac{\dot{A} \omega\left(\epsilon_{\mathrm{r}} \bar{r}_{0} \omega-R\right)+\left(2 \omega^{2} R+3 \omega^{3} \epsilon_{\mathrm{r}} \bar{r}_{0}+A \omega\right)\left(2 \epsilon_{\mathrm{r}} \bar{r}_{0}^{2} \lambda_{\mathrm{r}} \omega^{2} R^{-1}+\beta A+\lambda_{\mathrm{s}} R\right)}{2\left(2 \omega^{2} R+3 \omega^{3} \epsilon_{\mathrm{r}} \bar{r}_{0}+A \omega\right)+\left(\omega_{\mathrm{r}}^{* 2}-\omega^{2}\right)\left(\epsilon_{\mathrm{r}} \bar{r}_{0} \omega-R\right)},
$$

where $\omega=\omega(R)$ is given by $(52)$. Equations $(52,57)$ form a system that may be cast into the more compact form $\dot{R}=f[R, \omega(R)]$, which is in the form of a single nonlinear ordinary differential equation governing the slow modulation of the fast oscillations of the cylinder during the slowly decaying cycle. 


\section{Numerical results}

In this section, we validate the asymptotic analyses presented for the cases of small and large mass ratios by comparing the analytical predictions of the approximate solutions to numerical data obtained by directly solving the full infinite-dimensional fluidstructure interaction problem using Nek5000. We reiterate that, in the following results, we have set $\lambda_{\mathrm{s}}=0$, i.e., we have ignored viscous damping in the vertical support of the cylinder.

\subsection{Case of small mass ratio}

The response of the system obtained from direct numerical simulations for NES parameters $\epsilon_{\mathrm{r}}=0.05, \bar{r}_{0}=0.85$ and $\lambda_{\mathrm{r}}=0.054$ is shown in Fig. 2a. Frequency analysis is used to determine an approximate value of the detuning from the Strouhal frequency $\sigma$, and time series allow us to develop an ad hoc expression for the ansatz lift coefficient $\hat{C}_{\mathrm{L}}$. With this in hand, we use the asymptotic analysis developed in section 3 to predict the evolution of the system during a slowly-decaying cycle. In particular, we focus on one slowly decaying portion of the solution that develops from about 1200 to 1450 convective time units in Fig. 2a, and during which the NES locks in clockwise steady rotation caused by 1:1:1 resonance. We find $\sigma=-1.137$ to be an adequate value for the detuning parameter. The value of $\sigma$ is negative and an $\mathcal{O}(1)$ quantity, which is consistent with the definition of $\omega$ given earlier, and with the frequency analysis of Fig. 5, which shows that the dominant frequency of oscillations is slightly below the Strouhal frequency.

Using these ad hoc parameters, we extract from the data obtained by DNS an amplitude $N$ and a phase difference $\delta-\gamma$, and follow the trajectory in the corresponding phase-amplitude plane. Figure 8 depicts this trajectory, onto which the SIM described by (26) has been superimposed. Note that the trajectory is shown in a time interval going from 1140 to 1500 convective time units (and therefore spanning more than the time period of the considered slowly decaying cycle), which allows us to visualize the 1:1:1 transient resonance capture on the SIM and the ensuing escape from it. Indeed, the large-amplitude circulation of the trajectory at about $N=0.4$ corresponds to the early time response just before capture on the SIM. However, as the 1:1:1 resonance settles in, this transient circling pattern shrinks, as the dynamics is attracted on the SIM, and the trajectory "slides down" on the stable branch of the SIM. We note that, in theory, this "sliding" on the SIM might be arrested if the super-slow dynamics on the manifold were attracted by a stable attractor on the SIM, e.g., a stable fixed point. In the absence of such a stable attractor on the SIM, the "sliding" of the dynamics continues until the trajectory escapes the SIM and transitions into intermittent chaos. Hence, when the amplitude $N$ reaches a certain threshold — which appears to be reasonably close to the value predicted by $(27)$ — the system leaves the SIM, escape from resonance capture occurs, and the dynamics enters an intermittent chaotic regime where the cylinder response, the rotation of the internal NES and the flow field surrounding the cylinder (as evidenced by the lift force) become chaotic. This escape from resonance capture can be seen from the much more wriggly trajectory path in Fig. 8.

After a brief chaotic regime, the dynamics is again captured on the stable branch of the SIM, and the previous cycle of slowly decaying oscillation of the cylinder and uniform rotation of the NES starts anew. Physically, this means that, moments before escape from the SIM, the NES can no longer dissipate energy extracted from the cylinder 
motion, as it has been almost completely suppressed. When the dynamics leaves the SIM, the cylinder is energized by the flow, resulting in an increase of its oscillation amplitude. The system can then again be captured into the SIM, onto which it "slides" until escape. This cyclic pattern of captures into the SIM interrupted by non-resonant chaotic motions repeats itself endlessly. Similar dynamical behavior has been extensively described in the case of a vibro-impact NES attached to a linear oscillator subject to harmonic forcing [17], and compared to other topologically different SIMs [16, 37]. Considering the present problem, this result clarifies the nonlinear dynamical mechanism governing the repetitive cycles for small mass ratios depicted in Figs. 2(a, c, e) and 5(a, c, e, g).

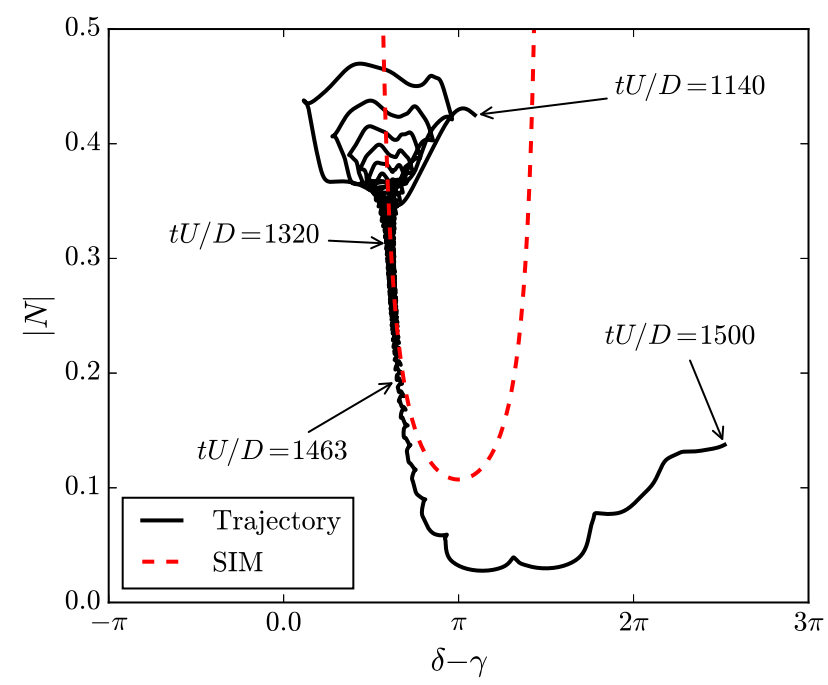

Figure 8: Capture into the 1:1 slowly invariant manifold, for NES parameters $\epsilon_{\mathrm{r}}=0.05, \bar{r}_{0}=0.85$ and $\lambda_{\mathrm{r}}=0.054$.

The approximate solution of the angular displacement of the pendulum shown in Fig. 9 accurately reproduces the response obtained by Nek5000 simulations during the slowly decaying cycle (ranging from 1200 to 1450 convective time units) and highlights the fact that the analytical approximation is valid only in the regime of 1:1:1 resonance, as evidenced by the poor agreement before resonance capture (for $t U / D<1200$ ) and after escape from the SIM (for $t U / D>1450$ ).

\subsection{Case of finite mass ratio}

For the case of finite mass ratio, we use NES parameters $\epsilon_{\mathrm{r}}=0.33, \bar{r}_{0}=0.458$ and $\lambda_{\mathrm{r}}=0.002745$ and propose an ansatz for the lift coefficient in the form of equation (47) that simulates the effect of the fluid on the cylinder. Comparison between the actual lift coefficient obtained by direct numerical simulation and the approximate single-frequency lift coefficient is shown in Fig. 10, and makes it visually clear that the higher harmonic components (here the third one) are not accounted for in the reduced-order model. Yet, as shown below, this approximation does not affect the efficacy of the analytical predictions. 


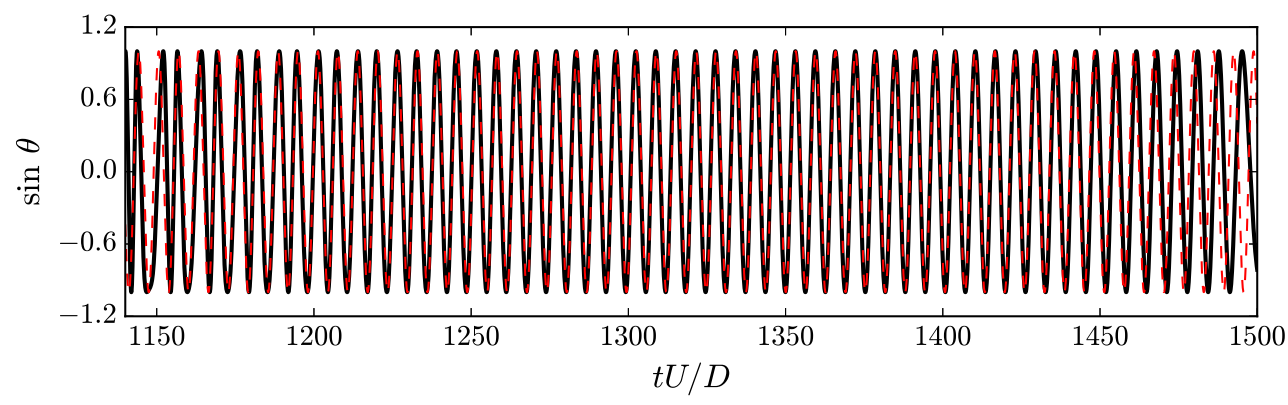

Figure 9: Sine of the angular displacement obtained by direct numerical simulation (solid) and its approximate solution (dashed) during capture into the 1:1 slow invariant manifold, for NES parameters $\epsilon_{\mathrm{r}}=0.05, \bar{r}_{0}=0.85$ and $\lambda_{\mathrm{r}}=0.054$.
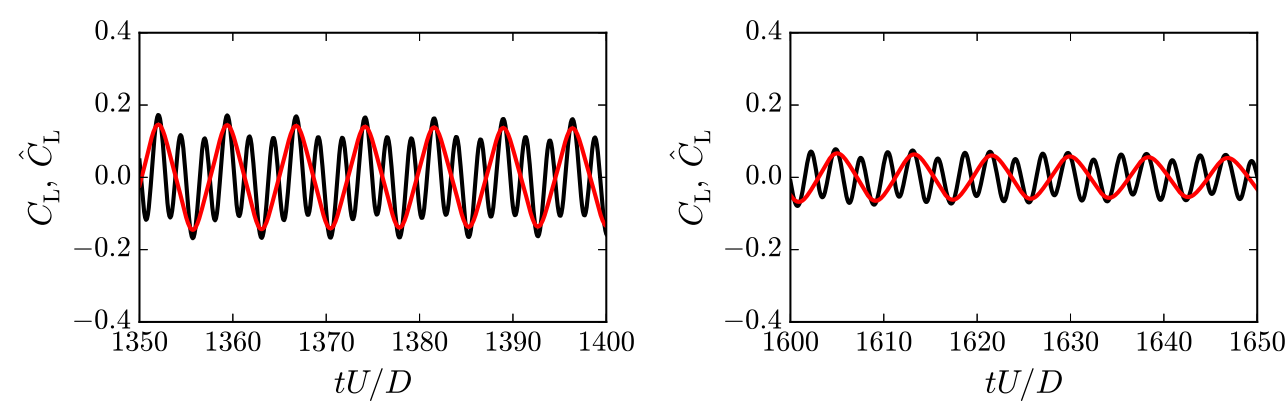

Figure 10: Lift coefficient $C_{\mathrm{L}}$ obtained by direct numerical simulation (solid black) and its monoharmonic ansatz $\hat{C}_{\mathrm{L}}$ (solid red) toward the beginning (left) and the end (right) of capture into the 1:1 slow invariant manifold, for NES parameters $\epsilon_{\mathrm{r}}=0.33, \bar{r}_{0}=0.458$ and $\lambda_{\mathrm{r}}=0.002745$.

We show in Fig. 11 that the frequency-amplitude relationship predicted by the theory in (51) agrees very well with the wavelet spectrum of the cylinder displacement computed with Nek5000. This figure was obtained by substituting (37) that relates the amplitude $R$ to the variables $y$ and $\dot{y}$ (which are directly computed by Nek5000) in (51), which yields a non-polynomial algebraic equation that can be solved for $\omega$ at each time step, given $y$ and $\dot{y}$ (this amounts to "fitting" the model to the raw data). Good agreement is also found between the angular displacement of the pendulum and its approximate solution (see Fig. 12).

Integration of the slow-flow equations subject to initial conditions extracted from the computational data yields an approximate amplitude $R$ that represents the envelope of the fast oscillations of the cylinder, as per (37). As shown in Fig. 13, the direct numerical simulation and approximate analytical solution agree well with each other. Finally, we show in Fig. 14 the "sliding" of the trajectories (both from direct simulation and from the approximate model) on the slow invariant manifold. Clearly, the slowly decaying cycle observed in the infinite-dimensional system is proven to be the result of the 1:1:1 resonance capture into the stable branch of the SIM. This capture is accompanied by 

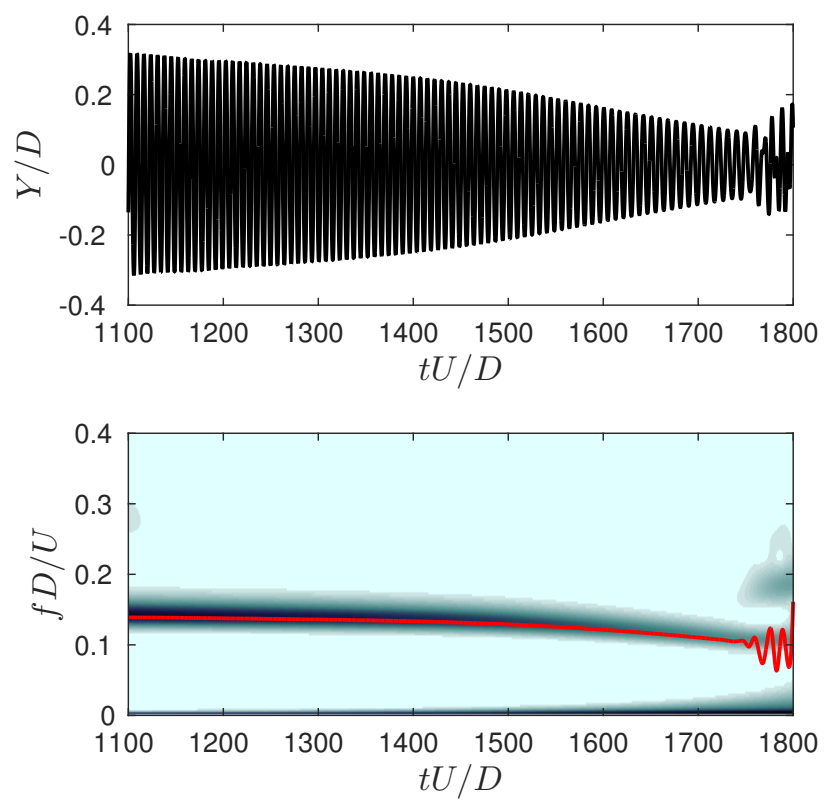

Figure 11: Time history (top) and wavelet spectrum (bottom) of cylinder displacement obtained by direct numerical simulation shown during capture into the 1:1 slow invariant manifold, for NES parameters $\epsilon_{\mathrm{r}}=0.33, \bar{r}_{0}=0.458$ and $\lambda_{\mathrm{r}}=0.002745$. The thick red line in the wavelet spectrum corresponds to the analytically predicted frequency-amplitude relationship (51).

a slow decrease in amplitude and frequency of the response of the sprung cylinder and, in the absence of any stable fixed points on the stable branch of the SIM, breaks down (at a value somewhat larger than the one predicted by (45) of the analytical model) when the amplitude $R$ becomes too small, following which the system escapes from the manifold and bursts into intermittent chaos, exactly as for the case of small mass ratios examined in the previous section. However, the important difference that distinguishes the 1:1:1 resonance capture for finite mass ratios is that, in this case, there occurs vortex street elongation and partial stabilization of the wake behind the oscillating cylinder. This important finding relates the resonant (nearly uniform) rotation of the internal NES rotator with the partial vortex stabilization of the wake. This interesting finding indicates that an internal nonlinear element can drastically affect the fluid flow past the oscillating cylinder, even though it is not in direct contact with it. Our analysis proves that, in the particular simulations considered here, the nonlinear mechanism of partial vortex stabilization is 1:1:1 resonance capture on the stable branch of the SIM.

\section{Concluding remarks}

We show that the mechanism of passive VIV suppression of a sprung cylinder restricted to move in the cross-flow direction and incorporating a strongly nonlinear internal dissipative rotator reported by Tumkur et al. [36] and characterized by successions 


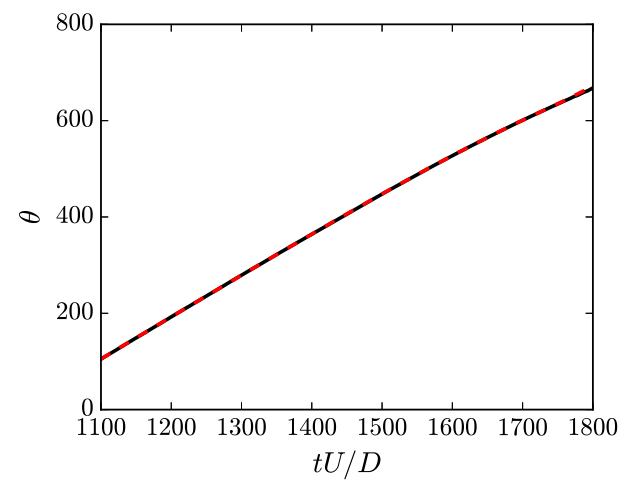

(a)

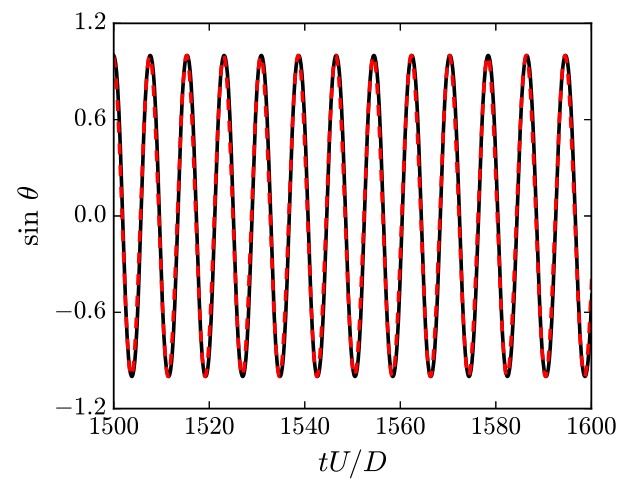

(b)

Figure 12: (a) Angular displacement and (b) its sine obtained by direct numerical simulation (solid) and approximate solution (dashed) during capture into the 1:1 slow invariant manifold, for NES parameters $\epsilon_{\mathrm{r}}=0.33, \bar{r}_{0}=0.458$ and $\lambda_{\mathrm{r}}=0.002745$.

of cycles of regular, slowly decaying motion interrupted by intermittent chaotic bursts, exists over a wide range of NES parameters at $R e=100$. We find that lower values of the mass ratio demand a stronger damping coefficient $\lambda_{\mathrm{r}}$ and a larger radius $\bar{r}_{0}$ for such a cycle to occur (and, in contrast, higher values of $\epsilon_{\mathrm{r}}$ require much weaker damping and a smaller radius), and observe qualitative differences in the response as well as in the wake behind the cylinder depending on the size of the mass ratio relative to other quantities and parameters.

When the mass ratio is small, the lock-in phenomenon between cylinder, pendulum and lift coefficient is governed by 1:1:1 resonance capture onto the stable branch of a slow invariant manifold (SIM), and is characterized by in-unison oscillations at a dominant frequency that remains close to the Strouhal frequency. Assuming an adequate expression for the surrogate lift coefficient (which may be extracted from numerical data based on the spectral-element solver Nek5000), the equations governing the motion of the resulting 2DOF system are averaged using scale-partitioning techniques, and the resulting slow flow becomes amenable to traditional multiple-scales asymptotic analysis with the mass ratio $\epsilon_{\mathrm{r}}$ being the small parameter of the problem. We derive the expression of the underlying SIM corresponding to a state of exact 1:1:1 resonance, and investigate the dynamics at the super-slow scale during transient capture on the SIM.

For larger values of mass ratio, the lock-in frequency common to the three subsystems (cylinder, pendulum and lift coefficient) slowly varies with time, and multiple-scales analysis is no longer applicable. We therefore resort solely to a complexification/averaging technique to unravel the topological structure of the SIM for the problem in this case. Along the lines of what was done for the case of small mass ratio, we study the superslow flow in the vicinity of the resonance manifold, and the transient capture followed by escape from the SIM. Comparison between direct numerical simulations and the derived analytical asymptotic results shows satisfactory agreement, thus demonstrating the efficacy of the asymptotic approach.

Comparing the two cases considered in this work (i.e., small and finite mass ratios), 


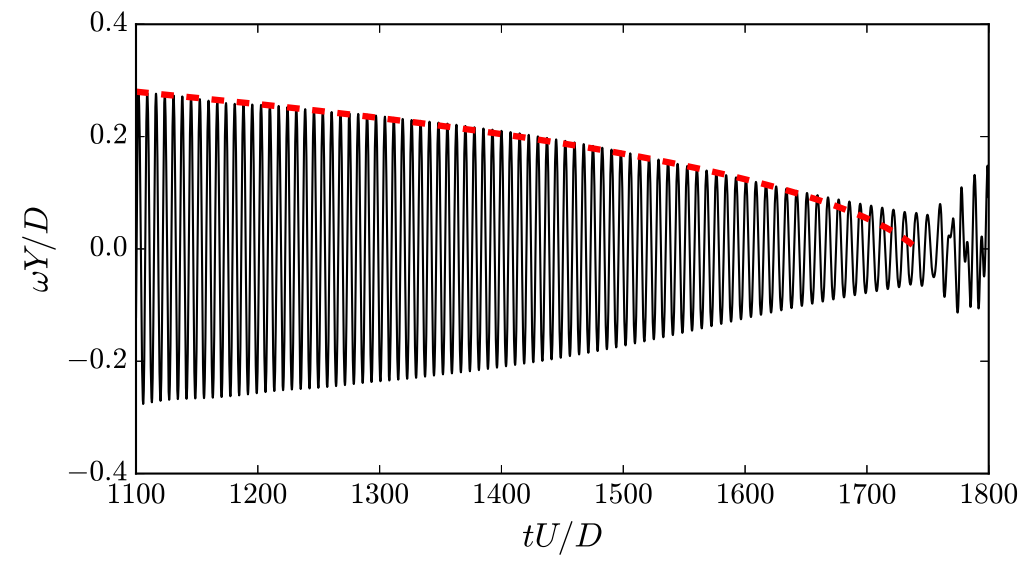

Figure 13: Time history of cylinder displacement (solid) and its approximate slow-flow solution obtained by integration of (57) (dashed) during capture into the 1:1 slow invariant manifold, for NES parameters $\epsilon_{\mathrm{r}}=0.33, \bar{r}_{0}=0.458$ and $\lambda_{\mathrm{r}}=0.002745$.

we note that the distinguishing feature of the transient nonlinear dynamics in the case of finite mass ratios is that, during resonance capture and the "sliding" on the stable branch of the SIM, there occurs significant vortex street elongation and partial wake stabilization in the wake of the cylinder. This indicates that resonance dynamics of an internal strongly nonlinear attachment inside a bluff body undergoing vortex-inducedvibrations can affect drastically (and even partially stabilize) the flow field behind it. In that context, it would be desirable to "insert" a stable attractor (e.g., a stable fixed point) on the stable branch of the SIM which could arrest the "sliding" of the orbit of the system during resonance capture and, hence, permanently (rather than intermittently as in the present case) stabilize the wake. This is the focus of current research on this topic.

\section{Acknowledgments}

The authors gratefully acknowledge use of the facilities at the National Energy Research Scientific Computing Center (NERSC). This work was supported in part by National Science Foundation (NSF) Grant CMMI-1363231.

[1] P. Argoul And T.-P. Le, Instantaneous indicators of structural behaviour based on the continuous Cauchy wavelet analysis, Mechanical Systems and Signal Processing, 17 (2003), pp. 243-250.

[2] V. I. Arnold, V. V. Kozlov, and A. I. Neishtadt, Dynamical Systems III. Encyclopedia of Mathematics, vol. 3, 1988.

[3] G. R. S. Assi, P. W. Bearman, N. Kitney, and M. A. Tognarelli, Suppression of wake-induced vibration of tandem cylinders with free-to-rotate control plates, Journal of Fluids and Structures, 26 (2010), pp. 1045-1057.

[4] A. Barrero-Gil, G. Alonso, And A. Sanz-Andres, Energy harvesting from transverse galloping, Journal of Sound and Vibration, 329 (2010), pp. 2873-2883.

[5] A. Barrero-Gil, S. Pindado, and S. Avila, Extracting energy from vortex-induced vibrations: a parametric study, Applied Mathematical Modelling, 36 (2012), pp. 3153-3160.

[6] P. Bearman, Circular cylinder wakes and vortex-induced vibrations, Journal of Fluids and Structures, 27 (2011), pp. 648-658. 

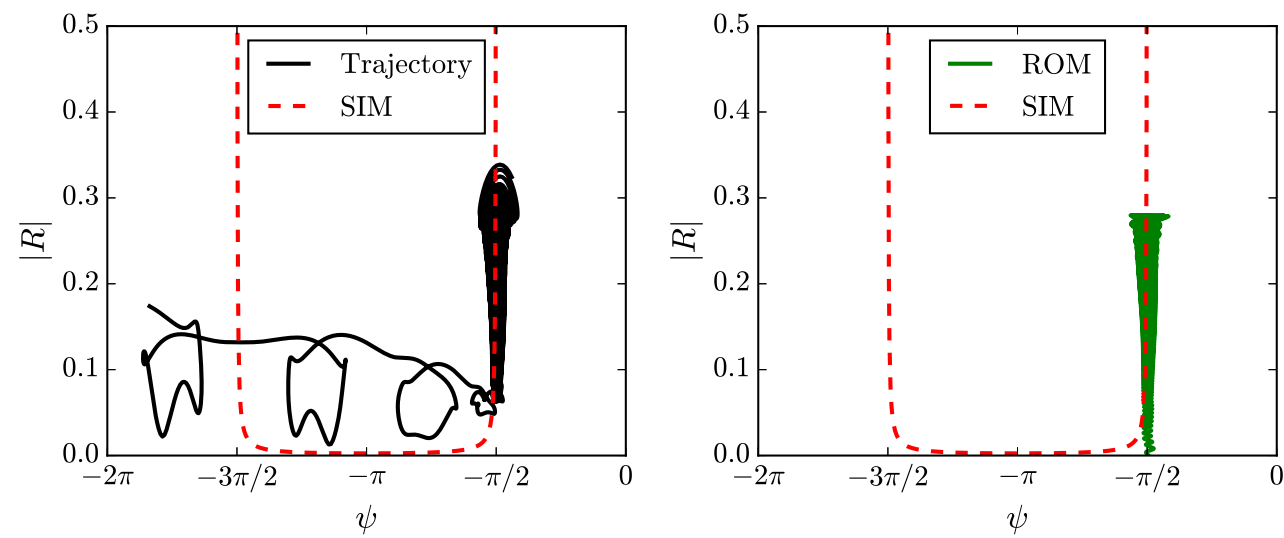

Figure 14: Capture into the 1:1 slowly invariant manifold, for NES parameters $\epsilon_{\mathrm{r}}=0.33, \bar{r}_{0}=0.458$ and $\lambda_{\mathrm{r}}=0.002745$. Comparison is shown between the raw data obtained by direct numerical simulation (left) and the analytical prediction resulting from integration of the reduced-order slow-flow model (ROM) described by (57) (right).

[7] P. W. Bearman, Vortex shedding from oscillating bluff bodies, Annual Review of Fluid Mechanics, 16 (1984), pp. 195-222.

[8] N. Benarous and O. V. Gendelman, Nonlinear energy sink with combined nonlinearities: Enhanced mitigation of vibrations and amplitude locking phenomenon, Proceedings of the Institution of Mechanical Engineers, Part C: Journal of Mechanical Engineering Science, 0954406215579930 (2015).

[9] M. M. Bernitsas And K. RAGHAVAn, Reduction/suppression of VIV of circular cylinders through roughness distribution at $8 \times 10^{3}<R e<1.5 \times 10^{5}$, in ASME 2008 27th International Conference on Offshore Mechanics and Arctic Engineering, American Society of Mechanical Engineers, 2008, pp. 1001-1005.

[10] M. M. Bernitsas, K. Raghavan, Y. Ben-Simon, and E. M. Garcia, ViVACE (Vortex Induced Vibration Aquatic Clean Energy): A new concept in generation of clean and renewable energy from fluid flow, Journal of Offshore Mechanics and Arctic Engineering, 130 (2008), p. 041101.

[11] M. O. Deville, P. F. Fischer, And E. H. Mund, High-order methods for incompressible fluid flow, Cambridge University Press, 2002.

[12] E. Domany and O. V. Gendelman, Dynamic responses and mitigation of limit cycle oscillations in Van der Pol-Duffing oscillator with nonlinear energy sink, Journal of Sound and Vibration, 332 (2013), pp. 5489-5507.

[13] M. L. Facchinetti, E. De Langre, And F. Biolley, Coupling of structure and wake oscillators in vortex-induced vibrations, Journal of Fluids and structures, 19 (2004), pp. 123-140.

[14] P. Fischer, J. Kruse, J. Mullen, H. Tufo, J. Lottes, And S. Kerkemeier, Nek5000 - Opensource Spectral Element CFD Solver, Argonne National Laboratory, Mathematics and Computer Science Division, Argonne, IL, (2008). See https://nek5000.mcs.anl.gov.

[15] R. Gabbai and H. Benaroya, An overview of modeling and experiments of vortex-induced vibration of circular cylinders, Journal of Sound and Vibration, 282 (2005), pp. 575-616.

[16] O. Gendelman, Targeted energy transfer in systems with external and self-excitation, Proceedings of the Institution of Mechanical Engineers, Part C: Journal of Mechanical Engineering Science, 225 (2011), pp. 2007-2043.

[17] O. V. Gendelman And A. Alloni, Dynamics of forced system with vibro-impact energy sink, Journal of Sound and Vibration, 358 (2015), pp. 301-314.

[18] O. V. Gendelman, A. F. Vakakis, L. A. Bergman, and D. M. McFarland, Asymptotic analysis of passive nonlinear suppression of aeroelastic instabilities of a rigid wing in subsonic flow, SIAM Journal on Applied Mathematics, 70 (2010), pp. 1655-1677. 
[19] A. Grossmann And J. Morlet, Decomposition of Hardy functions into square integrable wavelets of constant shape, SIAM journal on mathematical analysis, 15 (1984), pp. 723-736.

[20] C. Grouthier, S. Michelin, R. Bourguet, Y. Modarres-Sadeghi, and E. De Langre, On the efficiency of energy harvesting using vortex-induced vibrations of cables, Journal of Fluids and Structures, 49 (2014), pp. 427-440.

[21] C. Grouthier, S. Michelin, and E. De Langre, Energy harvesting by vortex-induced vibrations in slender structures, in Proceedings of the ASME 2013 32nd International Conference on Ocean, Offshore and Arctic Engineering OMAE2013, Nantes, France, 2013.

[22] J. Guckenheimer And P. Holmes, Nonlinear oscillations, dynamical systems, and bifurcations of vector fields, Springer, Berlin and New York, 1983.

[23] R. T. Hartlen And I. G. Currie, Lift-oscillator model of vortex-induced vibration, Journal of the Engineering Mechanics Division, 96 (1970), pp. 577-591.

[24] N. E. Huang, Z. Shen, S. R. Long, M. C. Wu, H. H. Shih, Q. Zheng, N.-C. Yen, C. C. Tung, AND H. H. LIU, The empirical mode decomposition and the Hilbert spectrum for nonlinear and nonstationary time series analysis, in Proceedings of the Royal Society of London A: Mathematical, Physical and Engineering Sciences, vol. 454, The Royal Society, 1998, pp. 903-995.

[25] W. Iwan And R. Blevins, A model for vortex induced oscillation of structures, Journal of Applied Mechanics, 41 (1974), pp. 581-586.

[26] G. Kerschen, A. F. Vakakis, Y. S. Lee, D. M. McFarland, and L. A. Bergman, Toward a fundamental understanding of the Hilbert-Huang transform in nonlinear structural dynamics, Journal of Vibration and Control, 14 (2008), pp. 77-105.

[27] G. Kerschen, K. Worden, A. F. Vakakis, And J.-C. Golinval, Past, present and future of nonlinear system identification in structural dynamics, Mechanical systems and signal processing, 20 (2006), pp. 505-592.

[28] L. I. MANEVITCH, The description of localized normal modes in a chain of nonlinear coupled oscillators using complex variables, Nonlinear Dynamics, 25 (2001), pp. 95-109.

[29] A. H. NAYfeh, F. Owis, and M. R. HajJ, A model for the coupled lift and drag on a circular cylinder, in ASME 2003 International Design Engineering Technical Conferences and Computers and Information in Engineering Conference, American Society of Mechanical Engineers, 2003, pp. 1289-1296.

[30] J. C. Owen, P. W. Bearman, and A. A. Szewczyk, Passive control of VIV with drag reduction, Journal of Fluids and Structures, 15 (2001), pp. 597-605.

[31] G. Sighlov, O. V. Gendelman, M. A. Al-Shudeifat, L. I. Manevitch, A. F. Vakakis, and L. Bergman, Resonance captures and targeted energy transfers in an inertially-coupled rotational nonlinear energy sink, Nonlinear Dynamics, 69 (2012), pp. 1693-1704.

[32] G. Sigalov, O. V. Gendelman, M. A. Al-Shudeifat, L. I. Manevitch, A. F. Vakakis, and L. A. Bergman, Alternation of regular and chaotic dynamics in a simple two-degree-of-freedom system with nonlinear inertial coupling, Chaos: An Interdisciplinary Journal of Nonlinear Science, 22 (2012), p. 013118.

[33] R. K. R. TumkuR, Modal interactions and targeted energy transfers in laminar vortex-induced vibrations of a rigid cylinder with strongly nonlinear internal attachments, PhD thesis, University of Illinois at Urbana-Champaign, 2014

[34] R. K. R. Tumkur, R. Calderer, A. Masud, A. J. Pearlstein, L. A. Bergman, And A. F. VAKAKIS, Computational study of vortex-induced vibration of a sprung rigid circular cylinder with a strongly nonlinear internal attachment, Journal of Fluids and Structures, 40 (2013), pp. $214-232$.

[35] R. K. R. Tumkur, E. Domany, O. V. Gendelman, A. Masud, L. A. Bergman, And A. F. VAKAKIS, Reduced-order model for laminar vortex-induced vibration of a rigid circular cylinder with an internal nonlinear absorber, Communications in Nonlinear Science and Numerical Simulation, 18 (2013), pp. 1916-1930.

[36] R. K. R. Tumkur, A. J. Pearlstein, A. Masud, O. V. Gendelman, L. A. Bergman, And A. F. VAKAKIS, Intermediate Reynolds Number Flow past a Sprung Circular Cylinder with an Internal Nonlinear Rotational Dissipative Element. (submitted), 2016.

[37] A. F. Vakakis, O. V. Gendelman, L. A. Bergman, D. M. McFarland, G. Kerschen, and Y. S. LEE, Nonlinear targeted energy transfer in mechanical and structural systems, Springer Verlag, New-York, 2008.

[38] C. H. K. Williamson and R. Govardhan, Vortex-induced vibrations, Annual Review of Fluid Mechanics, 36 (2004), pp. 413-455. 